\title{
Ultrashort pulse generation in the mid-IR
}

\author{
H. Pires ${ }^{1}$, M. Baudisch ${ }^{1}$, D. Sanchez ${ }^{1}$, M. Hemmer ${ }^{1}$, J. Biegert ${ }^{2,1}$ \\ ${ }^{1}$ ICFO - The Institute of Photonics Sciences Mediterranean Technology Park 08860 \\ Castelldefels (Barcelona), Spain \\ ${ }^{2}$ ICREA - Institucio Catalana de Recerca i Estudis Avançats, 08010 Barcelona, Spain
}

\begin{abstract}
Recent developments in laser sources operating in the mid-IR $(3-8 \mu \mathrm{m})$, have been motivated by the numerous possibilities for both fundamental and applied research. One example is the ability to unambiguously detect pollutants and carcinogens due to the much larger oscillator strengths of their absorption features in the mid-IR spectral region compared with the visible. Broadband sources are of particular interest for spectroscopic applications since they remove the need for arduous scanning or several lasers and allow simultaneous use of multiple absorption features thus increasing the confidence level of detection. In addition, sources capable of producing ultrashort and intense mid-IR radiation are gaining relevance in attoscience and strong-field physics due to wavelength scaling of re-collision based processes. In this paper we review the state-of-the-art in sources of coherent, pulsed mid-IR radiation. First we discuss semi-conductor based sources which are compact and turnkey, but typically do not yield short pulse duration. Mid-IR laser gain material based approaches will be discussed, either for direct broadband mid-IR lasers or as narrowband pump lasers for parametric amplification in nonlinear crystals. The main part will focus on mid-IR generation and amplification based on parametric frequency conversion, enabling highest mid-IR peak power pulses. Lastly we close with an overview of nonlinear post-compression techniques, for decreasing pulse duration to the sub-2-optical-cycle regime.
\end{abstract}

Keywords: Mid-infrared, nonlinear crystals, difference-frequency generation, difference-frequency generation, optical parametric amplifiers

\section{Contents}

1 Ultrafast mid-IR laser sources: enabling tools for cutting-edge applications

\footnotetext{
${ }^{1}$ hugo.pires@icfo.eu
} 
$\begin{array}{|ll|}2 & \text { Semiconductor mid-IR sources }\end{array}$

2.1 Quantum cascade lasers . . . . . . . . . . . . . . . . 5

2.2 Lead-salt diodes $\ldots \ldots \ldots \ldots \ldots \ldots$

\begin{tabular}{|lll}
\hline 3 & Crystal gain storage based ultrafast mid-IR sources & 8
\end{tabular}

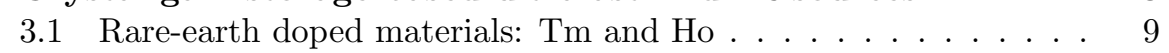

3.2 Transition metal chalcogenides: $\mathrm{Cr}$ and $\mathrm{Fe}$. . . . . . . . . . . 11

4 Parametric, non-gain-storage ultrafast mid-IR sources 12

4.1 Nonlinear crystals for the mid-IR . . . . . . . . . . . 13

4.2 Quasi-phase-matching . . . . . . . . . . . . . . . . . . . . . . . . 16

4.3 Difference-Frequency Generation . . . . . . . . . . . . . . . . 17

4.4 Optical Parametric Oscillators . . . . . . . . . . . . . . . . . . . 19

15

4.5 Optical Parametric Amplification . . . . . . . . . . . . . . . . . . 20

5 Mid-IR nonlinear spectral broadening and nonlinear compression 21

5.1 Nonlinear spectral broadening in the mid-IR . . . . . . . . . . 21

5.2 Nonlinear self-compression techniques in the mid-IR . . . . . . 22

$20 \quad 6$ Conclusions 223

\begin{tabular}{lll}
\hline 7 & Acknowledgments & 24
\end{tabular}

$\begin{array}{lll}8 & \text { References } & 24\end{array}$

\section{Ultrafast mid-IR laser sources: enabling tools for cutting-edge ap- plications}

25 Since the early days of the laser, mid-IR laser sources $(3 \leq \lambda \leq 8 \mu \mathrm{m})$ have been highly relevant for a number of applications, for spectroscopy in particular. Indeed, a large number of molecules and molecular functional groups feature both vibrational and rotational resonances in the mid-IR spectral region (see Fig. 1). For decades, spectroscopic applications such as absorption spectroscopy 30 or light detection and ranging (LIDAR) have driven the development of traditional laser sources in the mid-IR, typically operating in the continuous wave or nanosecond regime.

The emergence of transition ion doped-based laser oscillators (Ti:Sapphire, Cr:LiSAF, Alexandrite) in the mid-80s and their amplification in the early 90s

35 has triggered the dawn of ultrafast photonics in the visible spectral range with all the applications - even industrial - connected with these technologies. In the mid-2000s, it was recognized that the development of ultrafast - or equivalently ultra-broadband - laser sources in the mid-IR would address a number of shortcomings encountered at visible wavelengths. For instance - continuing

40 on spectroscopic applications [1] - the emergence of frequency comb sources would benefit from scaling to the mid-IR spectral range to tackle with record 


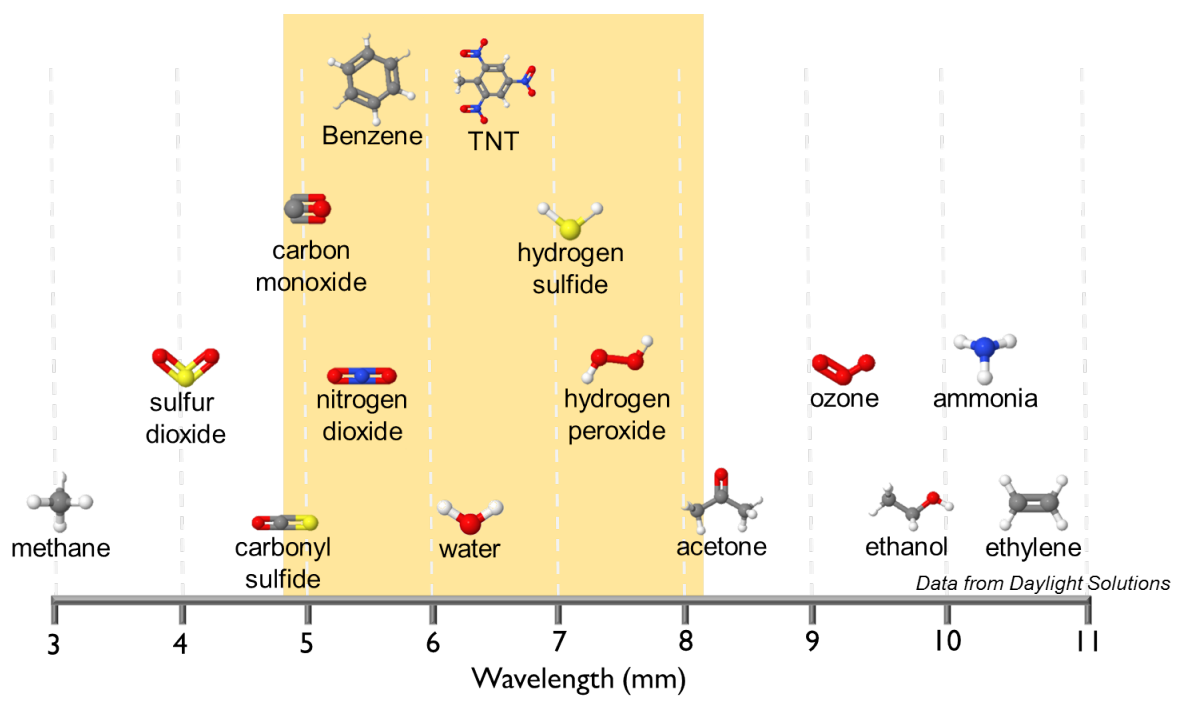

Figure 1: Molecular resonances in the mid-IR spectral region.

accuracy the numerous absorption lines present in the mid-IR spectral range. As mentioned above, over the past few decades, the field of mid-IR has made steady progress to refine sources yet, over the last decade, the field of ultrafast 45 mid-IR sources has spectacularly bloomed. It would therefore be far beyond this review to describe the various developments, their characteristics as well as applications. Hence, we restrict ourselves to the exciting domain of ultrafast mid-IR lasers. Even attempting to narrow our range of interest, the young field of mid-IR ultrafast sources and related applications is extremely diverse (Fig. 2): 50 mid-IR sources have great potential for extreme applications such as high order harmonic generation and attosecond pulse generation. Ultrashort pulses in the mid-IR permit scrutinizing atomic and molecular dynamics 2, 3, 4, they allow imaging molecular structure [5], and they enable scaling of coherent soft X-ray generation beyond the $\mathrm{keV}$ on a tabletop [6]. In medicine, mid-IR lasers permit 55 localized cutting and tissue removal due to the strong absorption of tissue at $6.45 \mu \mathrm{m}$ [7. Micrometer precision cutting and surgery are possible which minimize collateral damage and possible function loss. Longer wavelength sources also find industrial applications: at the edge of the near-IR, $2 \mu \mathrm{m}$ Thulium fiber lasers have been used to perform high efficiency high tensile strength polymer 60 welding, a clear improvement over welding with shorter wavelength sources 8 , and it is expected that as mid-IR ultrafast sources mature, they also will greatly benefit industrial technology. Military applications range from target designation to directed infrared counter-measures for jamming guidance systems 9 .

Figure 3 summarizes some of the main sources which are based on semi65 conductor and solid-state gain media as well as parametric processes, capable of operating in and between the two high atmospheric transmission windows 


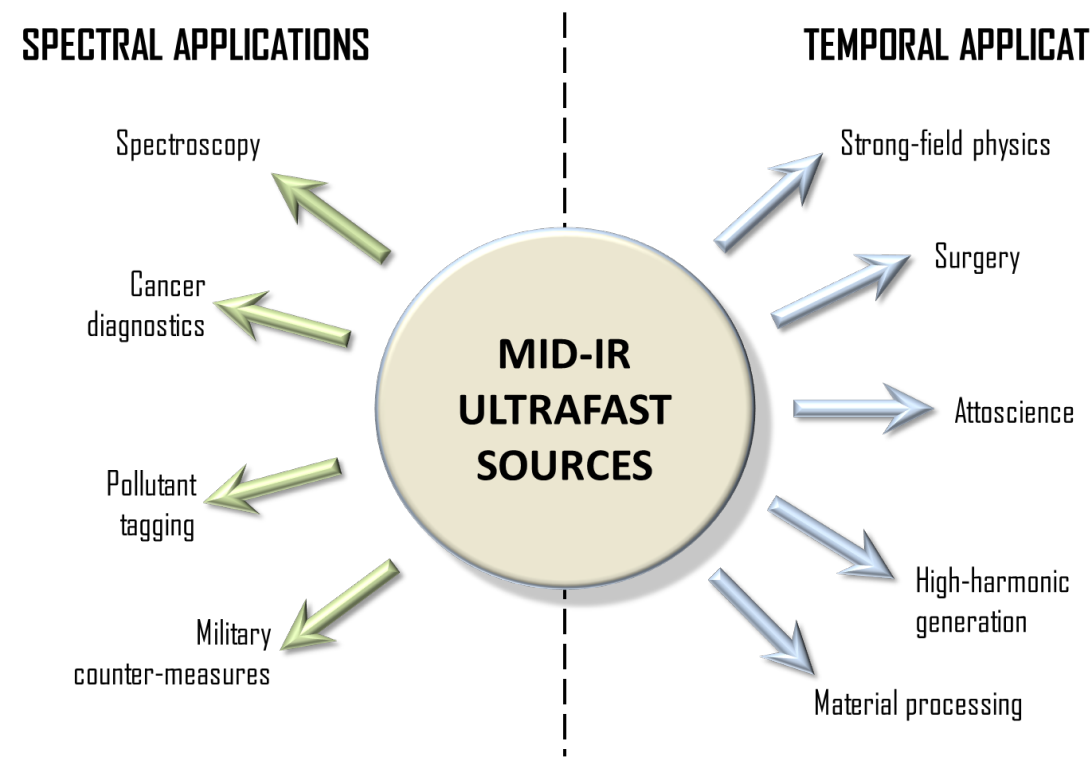

Figure 2: Mid-IR ultrafast sources applications.

(large absorption interval occurs from 5-8 $\mu \mathrm{m}$, so two transparency regions can be considered, at 3-5 $\mu \mathrm{m}$ and $8-14 \mu \mathrm{m}$ ).

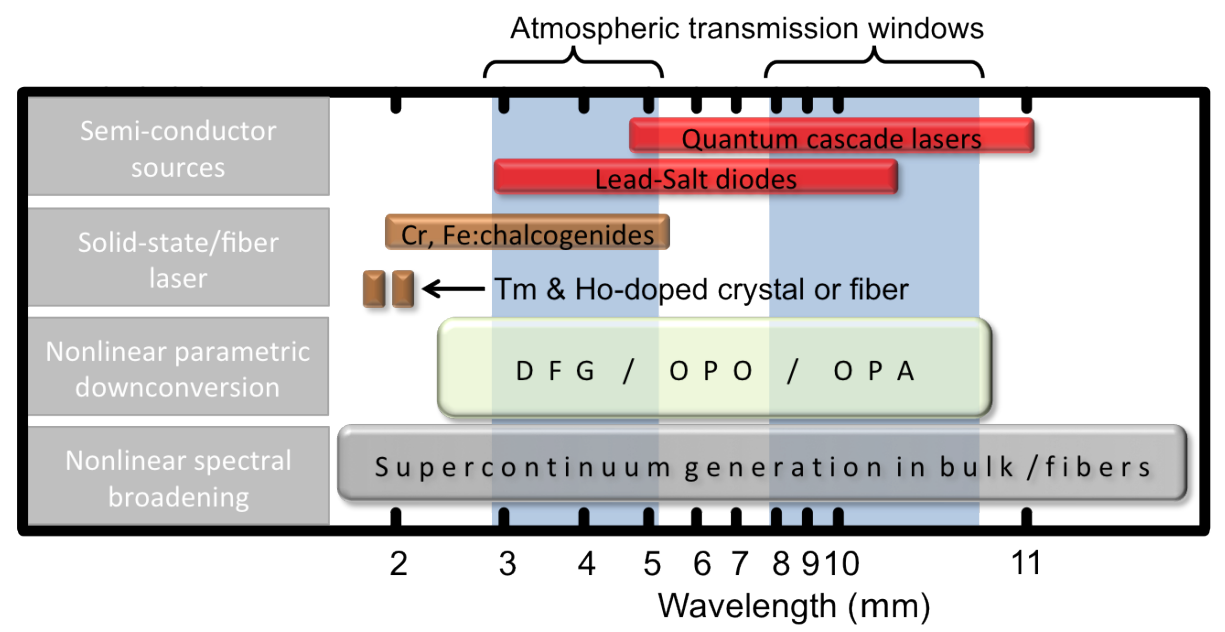

Figure 3: Laser sources and typical wavelength coverage. Shown are also the wavelengths of the two atmospheric windows [1].

There is a wide array of approaches to generate coherent long wavelength 70 light, with many gain materials, be it through frequency conversion or coherent shifting in liquids, gases and solids and we cannot do justice to all these fantas- 
tic solutions. Throughout the review we will identify the most well established and/or promising laser sources/amplifiers, giving a very brief historical perspective, detailing important characteristics and presenting the state-of-the-art as well as an outlook. The main contenders for direct electrical to optical energy conversion in the mid-IR spectral region are quantum cascade lasers (QCL) or lead-salt semiconductor lasers. These sources are discussed in the first section of this review. Traditional solid-state laser based-solutions relying on Chromium ion $\left(\mathrm{Cr}^{2+}\right)$-doped bulk to transfer optical energy from the visible or near-IR into

so the 2-5 $\mu \mathrm{m}$ range along with Thulium (Tm) and Holmium (Ho) doped materials are then discussed in Sec 2 of this review. The particular relevance of Tm and Ho doped solid-state lasers as pump for nonlinear parametric down-converters allows us to transition to the third section of this review in which differencefrequency generation, optical parametric amplification and optical parametric oscillation-based solutions are discussed as sources of mid-IR ultrashort pulses. Lastly we review the promising field of mid-IR nonlinear broadening and compression techniques, allowing the generation of multi-octave spanning few-cycle pulses.

\section{Semiconductor mid-IR sources}

Semiconductor technology is of two-fold importance for laser systems. On one hand semi-conductor lasers are compact, efficient direct optical energy generators, on the other hand they represent good pump sources for lasers. We discuss the two main semiconductor lasers operating in the mid-IR: quantum cascade lasers, that have been used to generate broadband few picosecond du-

95 ration laser pulses, and lead-salt diode lasers, whose integration in thin-disk architecture has enabled room temperature pulsed operation.

\subsection{Quantum cascade lasers}

Developed in 1994, QCLs are semiconductor lasers where the laser transition occurs between inter-sub-bands [10]. QCLs consist of a periodic series of thin

100 layers of different composition semiconductors, yielding an electric potential gradient over the thickness of the device. Therefore, the transiting electron, after leading to photon emission can tunnel into the next quantum well and undergo emission once more. Thus, the underlying principle of QCLs allows for each electron to generate as many photons as the number of stages in the setup, see Fig. 4. The emitted frequency by these devices is band-gap independent, and is determined by the thickness of the quantum wells, this being the reason for their large versatility. Also the carrier lifetime is in the picosecond range and thus the emission response can be directly modulated below the limit of normal semiconductor lasers.

Heat extraction is a concern at high power operation since heating lowers quantum efficiency and consequently the wall plug efficiency of only a few percent plagued the first generations of devices. Recent developments however allow for room temperature efficiencies of around $25 \%$ [12], or even $40-50 \%$ 


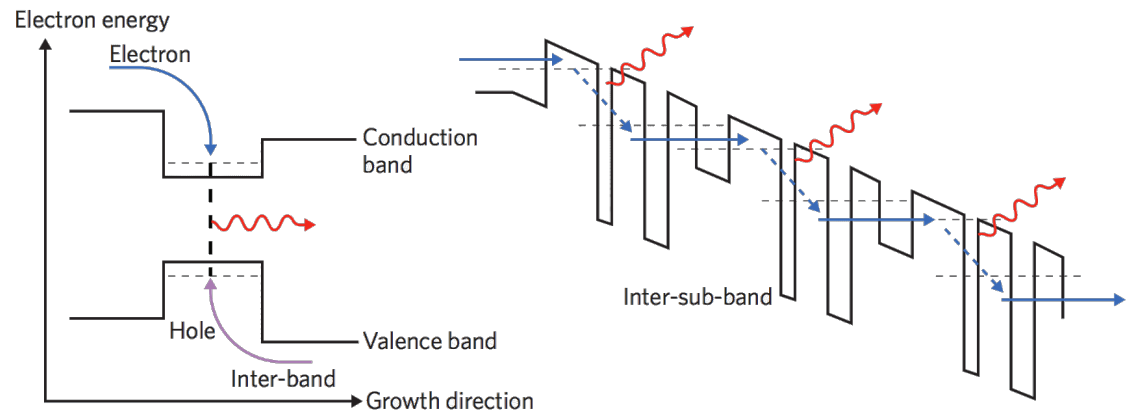

Figure 4: Schematics of inter-band laser and QCL (inter-sub-band) emission (left and right, respectively). Electron energy (y axis) shown versus growth direction of the device (horizontal axis). Valence band for the inter-sub-band structure is omitted [11].

with cryogenic cooling [13. Although there exist designs and techniques to create tunable QCL 14, their complexity and design requirements do not yet make them suitable for a turn-key oscillator. Similarly to diode lasers, their intrinsic beam divergence and astigmatism have to be compensated for, and power/energy is limited by currently available structure sizes. Recently, they have also been successfully fiber-coupled using hollow waveguides [15]

Inter-sub-band transitions lie normally in the terahertz $(\mathrm{THz})$ spectral region, however, new developments have enabled QCLs operating at short wavelengths down to $2.6 \mu \mathrm{m} \mathrm{[16}$, thus enabling access to vibrational levels in $\mathrm{C}-\mathrm{H}$ and $\mathrm{O}-\mathrm{H}(3-4.5 \mu \mathrm{m})[17,18$. They have also been mode-locked in a novel scheme [19] to generate picosecond pulses at the $\mathrm{THz}$ regime. QCLs can therefore operate throughout and even outside of the mid-IR spectral region, an achievement that makes them very attractive for compact, low power consumption (stand-alone, battery powered, for example) tools for trace-gas sensing and spectroscopy in general.

In the mid-IR, active mode-locking was obtained for 5 picosecond $8 \mu \mathrm{m}$ pulses at $11.6 \mathrm{GHz}$ [20. Attempts to passively mode-lock QCLs showed that the use of saturable absorbers is hindered by the fast gain recovery of the devices [21]. Furthermore, mode-locked QCLs have been used to create mid-IR frequency combs 222 and to create a compact, broadband and all solid-state device for dual-comb spectroscopy [23]. Active mode-locking has also been achieved lead-

135 ing to $3 \mathrm{ps}, 0.5 \mathrm{pJ}$ with wavelength at $6.3 \mu \mathrm{m}$ laser pulses [24], and a novel scheme for mode-locking was recently proposed: with an external ring cavity 25. the instabilities usually associated with mode-locked ultrashort QCLs are mitigated- an important step towards high power ultrashort QCLs.

\subsection{Lead-salt diodes}

140

Lead-salt materials have been widely used as photodiodes, due to their low band-gap allowing them to detect pulses throughout the mid-IR spectral region. They comprise a wide range of compound materials, namely, $P b_{1-x} S n_{x} S e$, 
$P b_{1-x} S n_{x} T e, P b S_{1-x} S e_{x}$ and $P b_{1-x} C d_{x} S$. Since the emitted wavelength in laser operation is given by the band-gap of the material, by adjusting the composition of these compounds, Lead-salt diodes can be tuned to operate from 3 to $30 \mu \mathrm{m}$. Similarly to near-IR and visible laser diodes, Lead-salt diode lasers suffer from considerable beam divergence and astigmatism, thus complicating optical designs for long distance propagation and/or tight focusing, as required by a number of applications.

150 A general issue for widespread applications of small band-gap materials is the requirement of cryogenic cooling to prevent thermal population of the conduction band. Therefore, despite their immense tunability allowing operation anywhere in the mid-IR, these practical constraints have prevented wide-spread use of mid-IR semiconductor lasers and led to a slow evolution of the technology.

155 However, among mid-IR semiconductors, lead-salt diodes have set the record for maximum operating temperature in both $\mathrm{CW}$ and pulsed regimes (as high as $60^{\circ}$ ) [26], thanks partly to much lower Auger recombination [27, compared to other mid-IR semiconductors (1-2 orders of magnitude lower than $\mathrm{HgCdTe}$, for example).

160 These materials have been integrated into Vertical Emission Cavity SurfaceEmitting Laser (VECSEL) architecture, allowing for lasing at $230 \mathrm{~K}$ in $\mathrm{CW}$ operation [28]. The VECSEL architecture has been shown to strongly reduce the beam divergence, allowing for single mode operation and lower threshold pump power operation, thus making them suitable for integration into mid-

165 IR gas sensing devices 29. More recently, VECSEL lasers operating at room temperature were built using a combination of narrow-gap PbTe and wide gap CdTe 30.

Lead salt materials have been used in multi quantum well 31 for pulsed operation, and in single quantum well microdisk lasers, also demonstrating $\mathrm{CW}$ 70 lasing at $275 \mathrm{~K} 32$.

This evolution in CW systems has also translated into a large improvement in pulsed operation: from the first implementations at cryo temperatures [33] up to even above room temperature [34, both pumped by neodymium ( $\mathrm{Nd}$ ) doped greater than 10 nanosecond pulse duration. Room temperature operation with

175 100-femtosecond optical pumping has also been reported 35] as well as pulsed operation at $4.4 \mu \mathrm{m}$ in a VECSEL using a PbSe quantum well [36, although reduced bandwidth from the latter would only support multi-picosecond pulses. Additionally, $8 \mu \mathrm{m}$ emission from VECSELs has been obtained in a cryo-cooled setup [37] in both CW and pulsed operation when pumped by a 360 nanosecond laser).

\section{Crystal gain storage based ultrafast mid-IR sources}

The longest wavelength solid-state laser reported to date operated at 7.2 $\mu \mathrm{m}$ wavelength, 38] relied on a $\mathrm{Pr}^{3+}: \mathrm{LaCl}_{3}$ crystal and was operated only in the pulsed regime at cryogenic temperature. The crystal used for this one-of-a185 kind laser was so hygroscopic that it had to be kept in a dry atmosphere with a dew point at $-40^{\circ} \mathrm{C}$ to maintain transparency over an extended period of 
time [39]. This example - though extreme - summarizes most of the challenges encountered when attempting to develop a mid-IR solid-state laser: materials exhibit extremely short excited-state lifetime, thereby requiring pulsed pumping, three-level operation, cryogenic cooling to control the thermal population of the intrinsically close-by energy levels, or difficulties to encounter materials with low phonon energy. For these reasons, only a handful of gain media have been reported to successfully operate in the mid-IR spectral range [40, 41, 42, 43, 44, 45.

$195 \quad$ Strictly speaking, the $2-3 \mu \mathrm{m}$ spectral region does not belong to the mid-IR spectral range. Nonetheless, a number of laser gain media exhibit transitions that have been successfully exploited in Q-switched and mode-locked operation. These "near-mid-IR" laser systems are highly relevant to pump parametric down-converters (see Sec 4) into the mid-IR and are therefore discussed below.

The gain media that have been successfully operated in the $2-3 \mu \mathrm{m}$ spectral range can be split into two families: rare-earth doped oxides or fluorides and transition metal doped chalcogenides. More specifically, the rare-earth doped materials most commonly used in the 1.9-2.1 $\mu \mathrm{m}$ spectral region are Tm or Ho-doped YAG, YLF and LuLF, see Fig. 5 a. Notice that some transitions 205 in Er-doped oxides also result in laser emission in this spectral range, but will not be discussed here since we aim at highlighting novel advances. More exotic sesquioxide doped laser gain media such as Lutetium oxide ( $\mathrm{LuO})$, Scandium oxide $\left(\mathrm{ScO}_{3}\right)$, Yttrium oxide (YO) and possibly compounds such as Lutetium Scandium oxide $(\mathrm{LuScO})$ show great potential - particularly in their ceramic form [46, 47] - when doped with $\mathrm{Yb}$ ions [48, 49, 50, and are expected to make an impact in the long wavelength range when doped with either Tm or Ho ions [51, 52, 53. The transition metal doped chalcogenide family consists in $\mathrm{Cr}^{2+}$ and $\mathrm{Fe}^{2+}$-doped ZnSe, ZnS, ZnTe, CdS and CdSe and emits in the 2.5 to $5 \mu \mathrm{m}$ spectral range, see Fig. $5 \mathrm{p}$. Notice that the $5 \mu \mathrm{m}$ boundary is only reached in ${ }_{215} \mathrm{Fe}^{2+}: \mathrm{ZnSe}$ and often requires drastic cooling - yet a lot of ongoing work aims at alleviating this cooling requirement - and pulsed operation [54, 55, 56.
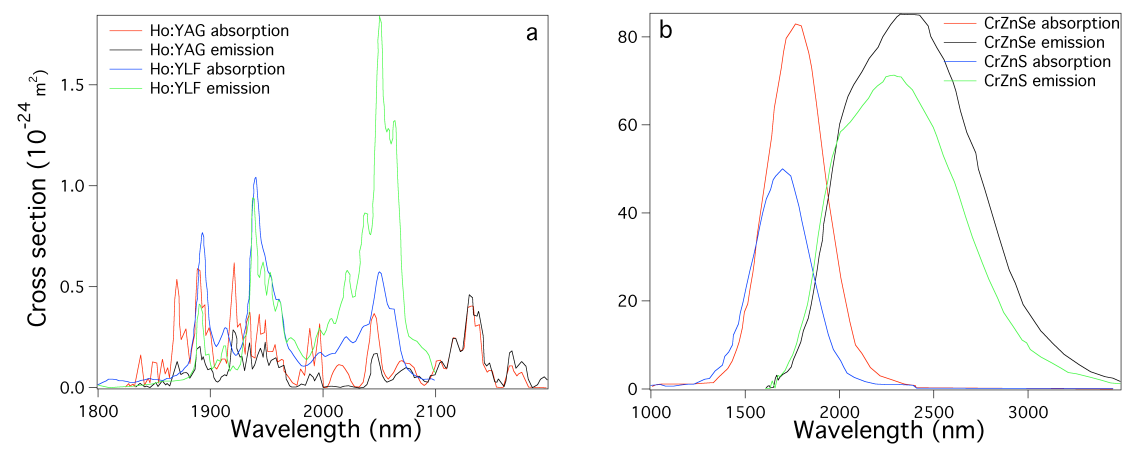

Figure 5: Chromium (a) and Holmium (b) absorption and emission spectra for different host materials. 
It is remarkable that Ho and Tm-doped oxides and fluorides have been investigated since the early days of the laser - as early as 1962 for a cryogenically cooled $\mathrm{Ho}_{\mathrm{CaWO}}$ [57] laser and 1965 for the first demonstration of room temperature operation of a Ho:YLF laser 58 - while transition metal doped chalcogenides - even though investigated since the 1960s [59, 60, 61, 62, 63, 64, 65, 66, 67. - were only produced with sufficient quality, and made to lase, in the mid 1990s at Lawrence Livermore National Laboratory 68. The first lasing action in $\mathrm{Cr}^{2+}$ :CdSe was reported in 200769 and complete spectroscopic 225 characterization of $\mathrm{Cr}^{2+}$ :CdS has been reported only this year [70. Cr:ZnTe is for the moment left rather unstudied [71. Both rare-earth doped and transition metal doped materials have recently experienced a revolution.

\subsection{Rare-earth doped materials: Tm and Ho}

Rare-earth doped oxide and fluoride materials have been used extensively in the 1990s, but suffered from the lack of a suitable and efficient pump laser. In the case of Tm ion doped materials, efficient absorption is possible in the red part of the visible spectrum $(790 \mathrm{~nm})$ with good overlap for pumping with GaAlAs diodes that are also used for pumping Nd:YAG, see Fig. 6. Using this efficient pumping channel to obtain emission in the $2 \mu \mathrm{m}$ spectral range nonetheless

235 results in low quantum efficiency - typically less than $40 \%$ - ultimately yielding heavy thermal loading of the crystals and preventing high power operation. A cross-relaxation process dubbed "2-for-1" 72 would in principle enable Tmdoped gain media pumped at $790 \mathrm{~nm}$ and emitting at $2 \mu \mathrm{m}$ wavelength to operate with a quantum efficiency as high as $80 \%$, but requires high dopant 240 concentration, a challenging task in a crystalline structure. This limitation was readily addressed with the rise of the fiber laser since heavy doping is more readily achieved in amorphous (glass) than crystalline matrices: Tm-doped fiber lasers make full use of the "2-for-1" process. Tm:fiber lasers have been reported to achieve efficiencies as high as $72 \%$ [73] when pumped at $1.6 \mu \mathrm{m}$ wavelength 245 and recently efficiency above $90 \%$ have been reported using resonant pumping 74. Most importantly, successfully porting high concentration of Tm ions into a glass matrix that is drawn as a fiber allows efficient cooling and has resulted in the delivery of continuous wave multi-kW level output powers in the $2 \mu \mathrm{m}$ spectral region [75]. In fact, Tm-doped fiber lasers have shown a 250 development similar to that of Yb-doped fibers in the last 15 years, see Fig.7. Continuous wave, narrowband tunable Tm-doped laser systems operating in the hundred Watt regime have been reported [76] and used to perform spectroscopic measurements over kilometer distances [77]. Tm-doped laser system have also been successfully Q-switched to drive nonlinear parametric down-converters 78 ,

255 79, 80. In addition to being highly relevant for high power CW operation and suited for Q-switched operation, Tm-doped fiber lasers - contrary to Tm-doped crystals [81] - exhibit a broad and smooth emission spectrum [82] making those lasers highly suitable for mode-locked operation in the picosecond 83] and few hundreds of femtosecond regimes 84,85 .

Ho-doped oxide and fluoride crystals have also been used extensively in the 1990s mostly through a co-doped Tm,Ho approach [87, 88, 89, 90, 91]. A ma- 

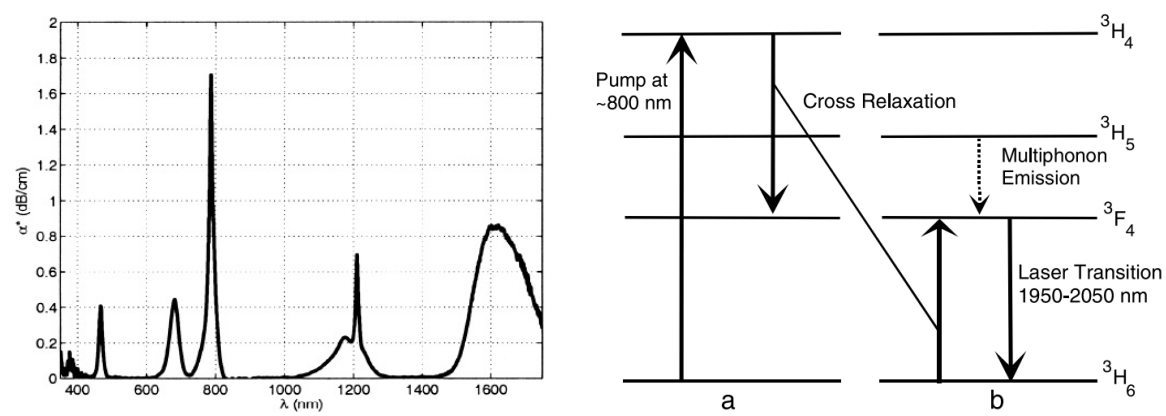

Figure 6: Spectral absorption of a Tm-doped single-mode silica fiber 86] (left) and Energy level diagram of two Tm ions (right), illustrating the cross relaxation process between them [72].

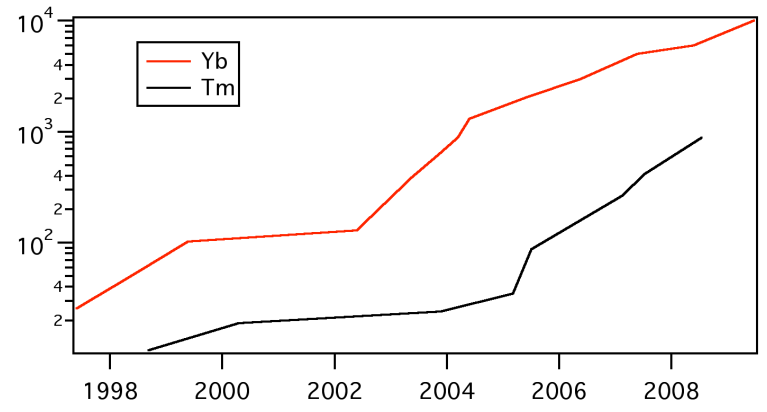

Figure 7: Power of $\mathrm{Yb}$ and Tm-doped CW fiber lasers plotted vs year.

jor hindrance for exploiting the full potential of the Ho ion was the need for a sensitizer - in this case Tm ions - to enable absorption at a wavelength where suitable pumps were available. The complex interactions between the Tm and Ho ions have been investigated 92 and proven to be the main limitation for power scaling of Ho, Tm codoped materials. The revolution occurred for Hodoped materials with the commercial availability of high power continuous wave Tm:fiber lasers which enabled decoupling energy transfer between the Tm and Ho ions. It is still early to summarize all the capabilities of Ho-doped materials 270 - partially plagued by a strong quasi-three level behavior 93 - but a number of exciting results have already been reported 94. A $1997 \mathrm{~nm} 410$ femtosecond near-transform-limited $84 \mathrm{~mW}$ passively mode-locked laser was reported 95. Fonnum et al. demonstrated a $0.5 \mathrm{~J}$, cryogenically cooled, nanosecond Q-switched Ho:YLF laser system operating at $1 \mathrm{~Hz}$ repetition rate 96. Malevich et al. reported a Ho:YAG CPA system operating at $5 \mathrm{kHz}$ and providing $3 \mathrm{~mJ}$ energy with pulses compressible to 530 femtoseconds [97. Recently, a 40 mJ, 10 picosecond CPA system operated at $100 \mathrm{~Hz}$ repetition rate relying on 
a water-cooled regenerative amplifier and cryogenically cooled single pass amplifier was developed [98, 99]. Direct 160 femtosecond output from a Ho-doped fiber oscillator, pumped by a commercial Tm-doped fiber laser has been implemented 100. The system delivers less than $1 \mathrm{~nJ}$ pulses at $35 \mathrm{MHz}$ and, using an additional nonlinear compressor stage, sub-100 femtosecond pulse durations were achieved. Hoogland et al. reported a $2.08 \mu \mathrm{m} \mathrm{Er:Tm,Ho} \mathrm{codoped} \mathrm{gain}$ fibers system, obtaining $7 \mathrm{MHz} 383$ femtoseconds (after compression) with an output pulse energy of $10.2 \mathrm{~nJ}$ [101.

\subsection{Transition metal chalcogenides: $\mathrm{Cr}$ and $\mathrm{Fe}$}

The present time is very exciting as a revolution for transition metal doped chalcogenides is currently occurring. Based on the smooth and broad emission cross-section exhibited by these materials 68, 102, 103, (see Fig. 8), it is clear that those gain media would be highly suitable for mode-locked operation. Mode-locking was achieved in Cr:ZnSe in 2006 using a saturable absorber and resulted in optical pulses with 100 femtosecond duration in the $2.5 \mu \mathrm{m}$ spectral range. These pulses have been further spectrally broadened in nonlinear fiber and yielded spectra spanning from $2000 \mathrm{~nm}$ to $2800 \mathrm{~nm}$ at $20 \mathrm{~dB}$ below the peak [104. Kerr-lens mode-locking was achieved [105, 106] and resulted in pulses as short as 95 femtoseconds at $94 \mathrm{MHz}$ repetition rate. This achievement resulted in the first frequency comb directly from a solid-state gain-stage based oscillator in a spectral region featuring numerous absorption lines from vibrational resonances in molecules.
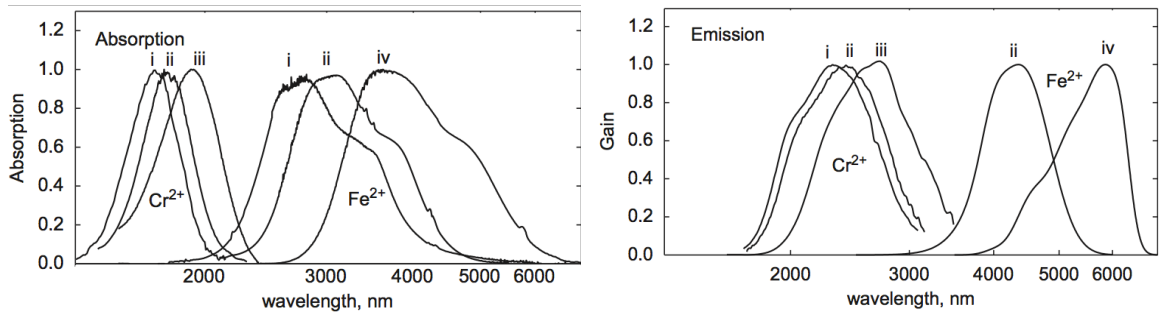

Figure 8: Absorption and emission cross-sections for several $\mathrm{Cr}$ and $\mathrm{Fe}$ doped materials: i:ZnS, ii:ZnSe, iii:CdSe, iv:CdMnTe [103.

Mode-locking operation in SESAMs and based on the Kerr effect in $\mathrm{Cr}^{2+}: \mathrm{ZnS}$ shortly followed in 2011 and 2013 [107, 108. It is questionable whether modelocked operation of Cr:CdS will be achieved owing to the short lifetime of the material at room temperature (480 ns) [70]. Mode-locked operation was shown in $\mathrm{Cr}^{2+}: \mathrm{CdS}$ at cryogenic temperature due to the lengthening of the excited 305 state lifetime $(7.8 \mu \mathrm{s}$ at $8 \mathrm{~K})$, but the technical requirement for such low temperatures might prevent widespread interest with $\mathrm{Cr}^{2+}$ :CdS. As can be seen from the very short time span over which all these exciting results have been reported, currently there is a tremendous effort to develop and perfect modelocked operation of these transition metal doped materials. This is the first 
ities of these transition metal doped laser systems, made possible by reliable Q-switched Ho-doped oscillators. Despite being plagued by relatively short lifetime, strong thermal lensing and high nonlinear refractive index, a CPA system based on $\mathrm{Cr}: \mathrm{ZnSe}$ that delivered $350 \mu \mathrm{J}$ energy at $1 \mathrm{kHz}$ with pulses as

315 short as 346 femtoseconds was recently reported by Slobotchokov et al. [109. The last aspect of the revolution currently ongoing for transition metal doped chalcogenide laser gain media lays in the implementation of these gain media in thin-disk geometry in an attempt to make full use of their remarkably high emission cross-section while mitigating the unfavorable thermal and nonlinear properties of the chalcogenide matrix [110, 111].

Similarly as with Fe-doped lasers, one of issues is the few suitable solid-state pump lasers, since their absorption region lies in the 3-4 $\mu \mathrm{m}$ range, efficient pumping can only be provided by a handful of solid-state lasers (Cr, Er-doped) or mid-IR semiconductors. The upper state lifetime and emission wavelength pulsed operation has yielded 15 nanosecond long $4.7 \mathrm{~mJ}$ at $4.3 \mu \mathrm{m}$ pulses with Fe:ZnSe pumped by a codoped Er:Cr:YSGG 20 nanosecond laser [56]. Just this year, a CW (Er:YAG) pumped Q-switched Fe:ZnSe laser was implemented which produced $0.5 \mathrm{~W}, 64$ nanosecond pulses [113. The luminescence study

330 from a QCL (80 kHz 100 nanosecond $4 \mu \mathrm{m})$ pumped Fe:ZnSe polycrystal has paved the way for high quantum efficiency, low phonon/thermal losses laser [114.

These developments clearly show the mid-IR solid-state laser panorama will see transition metal doped chalcogenides as main players and providers for high energy broadband, i.e. with short-pulse capability, especially when pumped by appropriate sources such as mid-IR semiconductors or rare-earth doped materials.

\section{Parametric, non-gain-storage ultrafast mid-IR sources}

In the previous section of this review we highlighted the challenges encountered when attempting to develop a mid-IR solid-state laser.

Difference-frequency generation (DFG), optical parametric generation (OPG) and optical parametric amplification (OPA) derive from the same set of nonlinear coupled wave equations. These equations are essentially frequency invariant and in principle valid for any wavelength range. In practice nonetheless, the

345 optical properties of the material in which the interaction takes place is of crucial importance. Several conditions such as transparency, absence of absorption, phase-matchability, absence of centro-symmetry and high nonlinearity must be met for a crystal to permit nonlinear interaction. One of the main characteristics of DFG, OPA and OPG arises from the three-wave nature of the interaction, supporting broad spectral coverage of the third wave.

Nonlinear three-wave mixing has been spectacularly successful to achieve frequency downconversion of solid-state lasers emitting in the visible or near-IR 
spectral range into the mid-IR region. In particular, the laser systems discussed in the previous section are expected to become major workhorses to drive such parametric down-converters well into the mid-wave and long-wave infrared. Three main nonlinear processes are used to achieve such frequency down-conversion: OPG, DFG and OPA. Notice that optical parametric amplifiers can be built resonantly and are then called optical parametric oscillator (OPO). All these processes are governed by the same set of three nonlinear coupled wave equations yet are used to fulfil different tasks.

Demonstrated in 1961, second harmonic generation (SHG) is often remembered as the first observed nonlinear process driven by a laser [115, this observation being achieved hardly a year after the invention of the laser itself [116]. It is less often remembered that the same year sum-frequency generation (SFG) 117 and optical rectification were also experimentally reported 118, laying down the building blocks that later enabled covering the electromagnetic spectrum emission from the UV to the $\mathrm{THz}$ through laser light frequency conversion. In this section of the review, we will mainly focus on DFG, OPA and OPO processes. These interactions were observed for the first time in 1963 for DFG [119]

370 and in 1965 for OPA [120, 121] and OPO [122. Owing to the narrow-spectral band of the lasers available in the early 1960s, all of these interactions led to small spectral bandwidth and little tunability.

\subsection{Nonlinear crystals for the mid-IR}

Despite the large number of known nonlinear crystals for OPA [123, given the stringent requirements listed above, only a limited number of crystals are suitable candidates for broadband generation in the mid-IR. Most standard nonlinear oxide crystals, extensively used in all areas of nonlinear optics and for which there exists a well-established growth technology, are limited in transparency range to around $5 \mu \mathrm{m}$ due to intrinsic multi-phonon absorption. Ex380 tending parametric processes to the entire mid-IR therefore requires turning to non-oxide materials such as binary and ternary non-centrosymmetric inorganic crystals. In general, these materials require more complex growth processes than oxides, which inevitably leads to an increase in growth defects and corresponding residual losses. Nevertheless, over the last two decades, an intensive search

385 for new nonlinear materials suitable for the mid-IR has led to an improvement on existing crystals as well as on the synthesis of new materials 124 .

A list of the most widely used or promising new mid-IR nonlinear crystals is shown in Tab. 1. Potassium titanyle arsenate (KTA) has been used to neartransform-limited 94 femtoseconds at $3.5 \mu \mathrm{m}$ with tens of $\mathrm{mW}$ [125] and to 390 generate $4.1 \mathrm{~W}$ of $3.5 \mu \mathrm{m}$ [126. An OPO with $31 \mathrm{~mJ}$ and $87 \%$ fundamental to mid-IR conversion efficiency was demonstrated [127]. Potassium titanyle phosphate (KTP) has been used to generate transform limited pulses in the 2.5-4.2 $\mu \mathrm{m}$ [128]; as well as a $250 \mathrm{kHz} 160$ femtosecond laser [129]. Potassium niobate (KNO) properties [130, 131] as a nonlinear crystal were investigated since the $39570 \mathrm{~s}$ [132, although its application for the mid-IR was considered only in the 90s [133]. It has been used to generate sub-5 cycle pulses at $3.6 \mu \mathrm{m}$ [134, sub 50 femtosecond pulses with $3-4 \mu \mathrm{J}$ at $3 \mu \mathrm{m}$ [135]. Of all potassium oxides its larger 
$\mathrm{d}_{\text {eff }}$, a large acceptance bandwidth and a higher damage threshold, make it attractive for energetic broadband operation [136]. Another group of oxide crystals are the ones involving lithium, namely lithium iodate, niobate and tantalum. Lithium niobate (LNO) has for long been used by the laser community, being a stable solution in the near-IR until the establishment of oxiborates (BBO, LBO) [122. When compared to these, its lower damage threshold coupled with presence of photorefractive effects limited power scaling. The photorefractive 405 effects can be controlled, however, by heating the crystal which changes the phase-matching conditions, or through $\mathrm{MgO}$ doping. Lithium tantalate has a broad transparency range (from UV to mid-IR), a high $\mathrm{d}_{\text {eff }}$ but regretfully exhibits very small birefringence, making it impossible to phase-match many of the desired configurations (SHG is not possible over the full visible region, for ${ }_{410}$ example, [137]). Lithium iodate has been used in an efficient DFG stage to produce $45 \mathrm{~mW}$ of $5 \mu \mathrm{m}$ pulses [138, and, having the longest transmission cutoff of the oxide crystals, it has also been used for mid-IR generation in the second atmospheric transmission window at $7 \mu \mathrm{m}$ [139. Silver thiogallate (AGS) and silver gallium selenide (AGSe) are negative uniaxial semiconductors that ${ }_{415}$ have been widely used for parametric downconversion to the mid-IR [140, 141. Their popularity stems from the fact that their large band-gap allows them to be pumped at $1 \mu \mathrm{m}$. AGSe has also proven to be an efficient up-conversion crystal for $\mathrm{CO}_{2}$ laser radiation 142 . However, both AGSe and AGS have low thermal conductivity and low damage threshold, making them unsuitable for high power application with femtosecond lasers.

\begin{tabular}{|l|c|c|}
\hline & Transparency range $(\mu \mathrm{m})$ & $d_{\text {eff }}(\mathrm{pm} / \mathrm{V})$ \\
\hline Oxide crystals & & 8 \\
\hline $\mathrm{KNbO}_{3}$ (KNO) & $0.4-4.5$ & 2.1 \\
\hline $\mathrm{KTiOAsO}_{4}(\mathrm{KTA})$ & $0.4-4.0$ & 2.3 \\
\hline $\mathrm{KTiOPO}_{4}(\mathrm{KTP})$ & $0.4-4.5$ & 2 \\
\hline $\mathrm{LiIO}_{3}(\mathrm{LIO})$ & $0.3-6.0$ & 4 \\
\hline $\mathrm{LiNbO}_{3}$ (LNO) & $0.3-5.5$ & 7 \\
\hline $\mathrm{LiTaO}_{3}$ (LTO) & $0.3-5.5$ & 23 \\
\hline $\mathrm{Non}-$ oxide crystals $^{\mathrm{A}}$ & & 41 \\
\hline $\mathrm{AgGaS}_{2}$ (AGS) & $0.5-1.3$ & 85 \\
\hline $\mathrm{AgGaSe}_{2}$ (AGSe) & $0.7-18$ & 54 \\
\hline $\mathrm{CdSiP}_{2}$ (CSP) & $0.7-9.0$ & 31 \\
\hline $\mathrm{GaSe}$ & $0.7-18$ & 10 \\
\hline $\mathrm{HgGa}_{2} \mathrm{~S}_{4}$ (HGS) & $0.5-13$ & 18 \\
\hline $\mathrm{LiGaS}_{2}$ (LGS) & $0.3-11$ & 10 \\
\hline $\mathrm{LiGaSe}_{2}$ (LGSe) & $0.4-13$ & 75 \\
\hline $\mathrm{LiInS}_{2}$ (LIS) & $0.4-12$ & \\
\hline $\mathrm{LiInSe}_{2}$ (LISe) & $0.5-12$ & \\
\hline $\mathrm{ZnGeP}_{2}$ (ZGP) & $2.0-11$ & \\
\hline
\end{tabular}

Table 1: Overview of nonlinear crystals for mid-IR parametric processes. 
Zinc germanium phosphide (ZGP) is a positive uniaxial chalcopyrite showing an extremely high nonlinearity [143] and it also presents good thermal conductivity as well as damage threshold. The transparency range is limited in the short wavelength part of the spectrum requiring a pump wavelength above 2 $\mu \mathrm{m}$ to avoid two-photon absorption (TPA). Nevertheless ZGP is one of the most widely used crystals for pumping mid-IR parametric devices and the most promising for the next generation of OPA based systems pumped at long wavelengths [144, 145.

Gallium selenide (GaSe) is a negative uniaxial crystal with a large nonlinearity and birefringence and has been extensively used for $\mathrm{THz}$ generation via intra-pulse DFG from Ti:Sapphire systems. Unfortunately, GaSe has a very low damage threshold and is an extremely soft crystal (0 on the Mohs hardness scale), which requires careful handling and makes it difficult to polish. In addition, it has a crystalline structure such that it can only be cut perpendicular to the optical axis, making certain phase-matching configurations unattainable [146.

The four ternary wide-band-gap biaxial crystals LGS, LGSe, LIS and LISe are relatively new additions to the list of mid-IR nonlinear crystals [147, 148, [149. Although LGS was reported as early as 1947, and poor quality sample of 440 LIS were studied in the 1970s, growth related problems prevented proper characterization of these materials until the last decade. They present the lowest nonlinearity of all the crystals considered here, around an order of magnitude less than ZGP for instance. On the other hand, their wide band-gap allows them to be directly pumped at $800 \mathrm{~nm}$ with femtosecond lasers without TPA 445 and their thermal properties, and damage threshold, are superior to the other mid-IR crystals like AGS and GaSe [150. Finally, Mercury thiogallate (HGS) and cadmium silica phosphate (CSP) are both promising new uniaxial chalcopyrites that have drawn increasing attention recently. HGS was originally developed in the 1970s [151 but growth problems limited the optical quality.

450 It presents a nonlinearity as high as AGS with a much higher damage threshold. The fabrication process has been greatly improved in the past decades and some practical application have been demonstrated [152]. CSP [153, 154] has the highest nonlinearity of all the crystals shown in this list [155] $\left(\mathrm{d}_{\text {eff }}=\right.$ $85 \mathrm{pm} / \mathrm{V}$ ) and can be pumped without TPA at $1 \mu \mathrm{m}$. Even though its relatively low damage threshold limits its application in high power systems, it has recently been proven to be a highly promising crystal for broadband mid-IR downconversion [156].

\subsection{Quasi-phase-matching}

In the case of some crystals, the crystal orientation required for phasematching leads to a low nonlinear coefficient value, making them undesirable. A solution to this problem is to orientate the crystal for maximum gain (use the largest element of the $\mathrm{d}$ tensor), and to use quasi-phase-matching (QPM) by having short periodic regions of the crystal, where the sign of the second order susceptibility alternates, so that after two consecutive regions the phase 
conditions are restored. In this case the parameter to be tuned to guarantee phase-matching is the thickness (or period) of each domain.

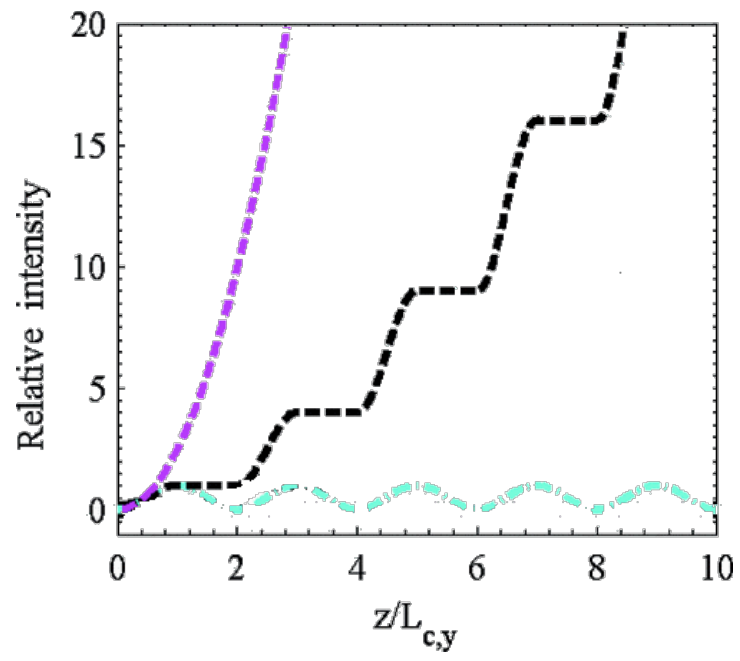

Figure 9: Example of gain versus propagation length for different types of phase-matching. The dashed purple line shows perfect phase-matching, dashed black line for perfect quasiphase-matching, dot-dashed cyan line for no phase-matching [157].

These materials are also called periodically poled or oriented, since their internal axis are inverted over every given period. One way of implementing this is using ferroelectric materials, where orientation of the extraordinary axis is imprinted through high electric field application at high temperatures.

Periodically poled Lithium Niobate (PPLN) is one of such ferroelectric oxides that has allowed extending OPAs from the near-IR into the mid-IR (up to the $5 \mu \mathrm{m}$ mark). Aperture increases, needed for energy scaling, have been steadily but slowly occurring; the difficulty lies in the scaling of the needed 475 voltages (kiloVolts even for few millimetres crystals) to pole thick crystals all the while also needing to maintain homogeneity and well defined poling locations (micrometer resolution) [158. Despite this, larger PPLN are being produced (for example $10 \mathrm{~mm}$ thick wafers have been successfully poled) as the fabrication technology develops, leading to the operation of a high efficiency half Joule level OPO [159].

Another way to quasi-phase-match is to periodically change the sign of the $\chi^{2}$ coefficient, this can be done by periodic inversions of the crystallographic orientation epitaxially grown into the crystal, called Orientation-Patterning (OP). Materials where this technique is used are GaAs, GaN and GaP. OP-GaAs is the most used so far, due to its large $d_{\text {eff }}, 1.5-15 \mu \mathrm{m}$ transparency range and a relatively small group velocity dispersion [160. It has been used to create milliwatt tuneable DFG outputs in the 7 to $13 \mu \mathrm{m}$ [161, see Fig. 10.

There are however some practical difficulties associated with size (and energy) scaling of OP crystals. Namely, the difficulty of having error free crystal 


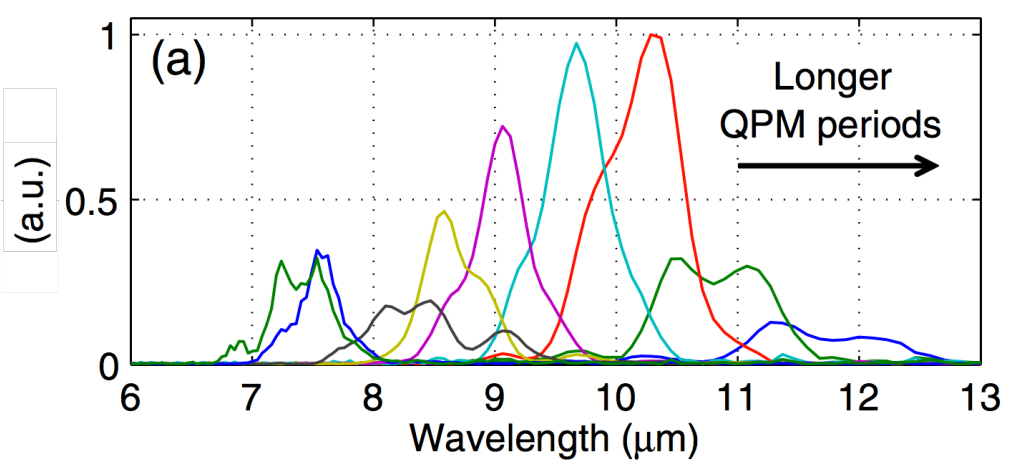

Figure 10: Mid-IR output spectra for different crystal periods [161]. (Maximum output power for the $10.3 \mu \mathrm{m}$ peak was $1.3 \mathrm{~mW}$ ).

inversions with poling thicknesses $10 \mu \mathrm{m}$ or less, limited OP-GaAs to be used in $10 \mu \mathrm{m}$ laser systems only. Due to the rapidly advancing technology, one can foresee that poling will be possible for bridging the $3-10 \mu \mathrm{m}$ spectral region.

\subsection{Difference-Frequency Generation}

Difference-frequency generation is highly attractive to generate broadband mid-IR radiation. The minimum requirements on the driving near-IR laser are modest as long as sufficient intensities are reached, i.e., typically requiring femtosecond pulses with energies as low as the nanojoule level. These specifications can be met by a number of solid-state or fiber laser sources, and allow for compact tuneable and broadband mid-IR sources [161. The DFG process was early

500 on implemented using two relatively narrow-band laser sources. Such implementation of DFG is often referred to as inter-pulse DFG, and still remains a robust way of down-converting near-IR laser systems into the mid-IR and at appreciable powers.

As an example, using a commercial fiber laser with two outputs tailored for DFG, an output of $1.6 \mathrm{~mW}$ at a repetition rate of $100 \mathrm{MHz}$ and with high quantum efficiency has been obtained [162] using Periodically Poled Lithium Niobate (PPLN). The spectrum spanned from 3-4 microns (at $\left.1 / e^{2}\right)$. Such spectrum can support CEP-stable 3 cycle pulses, and is ideal to seed a broadband mid-IR laser amplification system.

For $\lambda \geq 5 \mu \mathrm{m}$, CSP, has been used to generate a spectrum supporting 50 femtoseconds transform-limited duration pulses, (2.3 optical cycles) (Fig. 11 a). An average power of up to $15 \mathrm{~mW}(150 \mathrm{pJ})$ was obtained: AGS was also used for comparison and delivered $300 \mu \mathrm{W}(3 \mathrm{pJ})$ under the same conditions yielding less bandwidth, see Fig. 11b 163 .

${ }_{515}$ Additionally, LiSe has been used for DFG to generate nanojoules level energies tuneable from 5 to $12 \mu \mathrm{m}$, and up to $17 \mu \mathrm{m}$ with AGSe [164, 165, 166. With AGS and GaSe tunability between 5.2 to $18 \mu \mathrm{m}$ tunability was demonstrated [167, illustrating the flexibility of the DFG approach. 


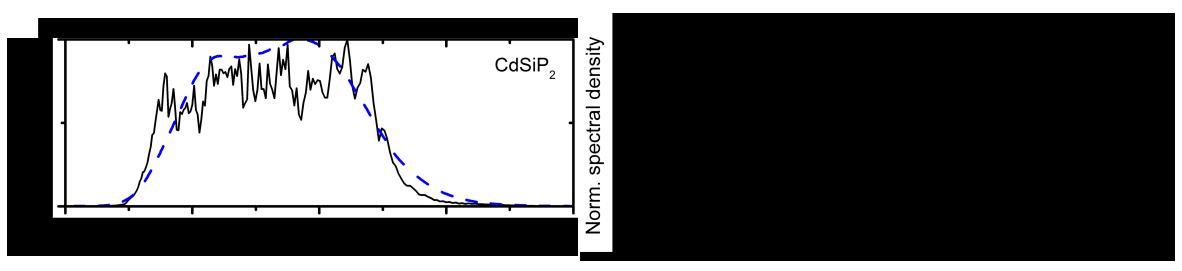

Figure 11: Measured idler spectrum (black solid line) together with simulated spectrum (blue dashed line) based on numerical simulations of the DFG process including absorption in $\mathrm{CaF}_{2}$. Left: CSP, Right: AGS [163.

Intra-pulse DFG [168] alleviates all issues related to spatio-temporal overlap of the iterating pump and signal waves but requires driving pulses featuring ultra-broad bandwidth. For instance generating $5 \mu \mathrm{m}$ radiation from a source centered at $800 \mathrm{~nm}$ requires significant spectral content at both $740 \mathrm{~nm}$ and 860 nm i.e. over 200 to $300 \mathrm{~nm}$ of spectral bandwidth. Until the mid-2000s the main drivers for intra-pulse DFG were Ti:Sapphire oscillators providing energy in the nanojoule to tens of nanojoules energy range. For instance, Jedju et al. reported on an ultrafast DFG setup based on a Ti:Sapphire laser at with spectral content at both $770 \mathrm{~nm}$ and $840 \mathrm{~nm}$ from the same oscillator 169 and successfully drove difference-frequency generation in an AGS crystal to the 7.5-12.5 $\mu \mathrm{m}$ range at $82 \mathrm{MHz}$ repetition rate and with $\approx 500$ femtoseconds pulse duration [170]. The development of thin-disk laser technology, and in particular mode-locked Yb:YAG thin-disk lasers delivering microjoule energy level directly from an oscillator, is currently changing revolutionizing the accessible parameter range and average power $[171,172,173,174,175$. While the spectral content typically found at the output of these oscillators is too narrow to drive DFG into the mid-

535 IR spectral range, the implementation of post-spectral broadening compression (in high throughput gas-filled hollow-core fiber and chirped mirror), results in pulses with duration in the few tens of femtosecond duration and energy in the microjoule range, highly suitable for driving intra-pulse DFG to the mid-IR 150. Limitations of intra-pulse DFG are nonetheless: (i) the efficiency of the process, which is typically limited since the same driving pulse serves as pump and signal for the interaction, (ii) the process offers limited adjustment and relies solely on temporal walk-off of the different spectral components within the pulse. Still, intra-pulse DFG enables stable temporal synchronization of the DFG stage input (and thus, stable spectral output), and allows for compact 545 octave-spanning systems in the mid-IR [150.

Another way of implementing mid-IR DFGs that requires a single laser is to couple some of the output into a fiber for soliton Raman shifting [176, 177, and then perform DFG between the Raman shifted and the fundamental pulse. This technique has been used to implement compact highly tuneable DFG [178, 179, 180. Another variant is based on the so-called adiabatic conversion; presented by Suchowski in 2008, it allows for ultra-high efficiency to take place in a short portion of the nonlinear crystal [181]. Recently, this technique has been used to 
down-convert the output of a Ti:sapphire system into the mid-IR with almost $100 \%$ efficiency [182, and generate an octave spanning mid-IR laser 183] with

\subsection{Optical Parametric Oscillators}

When resonantly pumped and placed inside a cavity, the parametric process is called optical parametric oscillation (OPO). This approach, owing to filtering of the cavity allows superior stability and tunability of the output.

560 ticular care. The quasi-instantaneous nature of the interacting pulses requires careful management of the intra-cavity dispersion and calls for short nonlinear crystals to achieve broadband generation, therefore limiting the gain per pass and the affordable output coupling losses.

Moreover, pumping with femtosecond driving pulses requires precise synchronization between the pump repetition rate and the cavity roundtrip.

The workhorse for mid-IR OPOs operated at wavelength shorter than $5 \mu \mathrm{m}$ has been periodically poled Lithium Niobate [184] for a number of years. For operation at longer wavelength, the negative uniaxial chalcopyrite AGS, AGSe 570 and positive uniaxial chalcopyrite ZGP have also been used in tandem OPOs [185, 186. Recently, the emergence of femtosecond Cr:ZnSe lasers permitted additional tandem OPOs to directly pump non-oxide nonlinear crystals in their transmission range. Notice that Ti:Sapphire [187, Er:fiber [188, Yb:fiber [189], Tm:fiber [190, have all been used to drive OPO into the mid-IR spectral range. 575 Advances in materials have also triggered exciting new possibilities in the field of mid-IR ultrafast OPOs: optically patterned GaAs has led to the first OPO featuring an isotropic material [160, see Fig. 12. The chalcopyrite crystal CSP has also been used in OPO for emission in the 5.8-6.6 $\mu \mathrm{m}$ spectral range by pumping at $1053 \mathrm{~nm}$. The output energy of $240 \mathrm{pJ}$ at $100 \mathrm{MHz}$ was nonetheless generate $27.5 \mathrm{~mW}, 6.45 \mu \mathrm{m}$ laser pulses, with the crystal performance being superior to the obtained with AGS [192].

A variety of OPO systems has been demonstrated with ZGP [144, 145, 193, [194, 195, 196, 197, yielding up to $15 \mathrm{~kW}$ peak power pulses at $3.7 \mu \mathrm{m}$ [80. This very year $200 \mathrm{~mJ}$ pulses (signal and idler) were obtained in the 3 to $5 \mu \mathrm{m}$ with high beam quality $\left(\mathrm{M}^{2}\right)$ using ZGP pumped by a Ho:YLF laser 198. A very successful implementation of OPO relies on a particular geometric arrangement, called RISTRA architecture [145, 198, 196, 199.

\subsection{Optical Parametric Amplification}

590

The aforementioned techniques and implementations are sufficient for spectroscopy and mid-IR low energy/power sources. Many applications however require high pulse energies. The process to amplify radiation is called optical parametric amplification, which comes in a number of varieties ranging from femtosecond OPAs to optical parametric chirped pulse amplifiers (OPCPA).

${ }_{595}$ Strictly speaking it is nearly impossible to generate unchirped pulses, we distinguish OPA from optical parametric chirped pulse amplification as OPCPA 


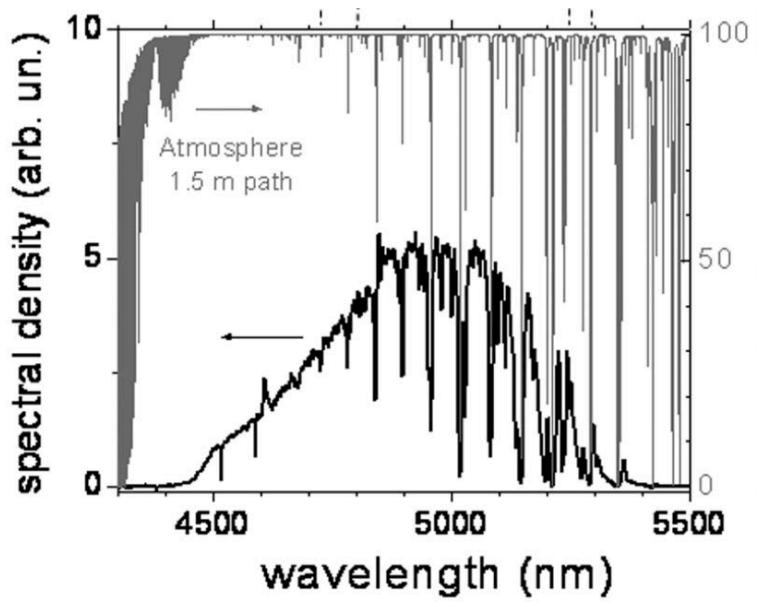

Figure 12: GaAs OPO output spectra (in black). Atmospheric absorption is shown in gray [160.

using intentionally chirped pulses compared to OPA. Broadband ultrafast OPAs operating at $2 \mu \mathrm{m}$ wavelength are commercially available from a number of vendors and are therefore of limited interest in this review. The most popular implementation is typically driven by high energy chirped pulse amplifier-type femtosecond Ti:Sapphire systems and are seeded by white-light sources. The parametric amplifiers in these cases use the ubiquitous $\beta$-Barium Borate (BBO) or Bismuth Triborate (BiBO) crystals, limiting operation to wavelengths shorter than $2 \mu \mathrm{m}$ due to the decreasing transparency of these crystals past $2 \mu \mathrm{m}$ wave605 length. A high-power home-built implementation of such femtosecond OPA in $\mathrm{BiBO}$ was reported by Silva et al. generating CEP-stable pulses at $2.1 \mu \mathrm{m}$ 200. Cascaded high-energy OPA-systems have nonetheless been implemented in which a second stage of parametric frequency conversion (mostly DFG) enables reaching the mid-IR spectral range at the expense of efficiency and sim${ }_{610}$ plicity 201, 202, 203. Maximum pulse energies up to $100 \mu \mathrm{J}$ at $4 \mu \mathrm{m}$ with 1 $\mathrm{kHz}$ repetition rate 202 could be demonstrated. In contrast, OPCPA enables order of magnitude further upscaling of the output energies. In the mid-IR, this technique has so far been implemented only in a handful of laboratories worldwide [204, 205, 206, 207, 208, but is gaining momentum particularly because ${ }_{615}$ of the impact in strong-field physics [2, 3, 6, 209. High power mid-IR outputs ranging from $18 \mu \mathrm{J}$ energy at $160 \mathrm{kHz}$ [205] up to $13 \mathrm{~mJ}$ energy at $10 \mathrm{~Hz}$ [206] could be demonstrated. The implementation of the mid-IR OPCPA systems are based on two routes for successful generation of high energy ultra-broadband optical pulses: 1)Parametric amplification of near-IR radiation at $1.3-1.6 \mu \mathrm{m}$ ${ }_{620}$ wavelength, and a resulting idler at either $3 \mu \mathrm{m}$ [207, 208, or $3.8 \mu \mathrm{m}$ [204, which is compressed and used, i.e. the last OPCPA stage is operated as DFG stage. This approach permits using easily available optics and components but on the expense of intrinsically carrier-envelope phase (CEP) stabilized pulses 
in the mid-IR. 2) The pathway followed by our group [205] places the DFG

stage in front, therefore providing intrinsic CEP stability if both DFG inputs originate from the same oscillator 210. This approach resulted in the delivery of passively and long-term CEP-stable optical pulses with 6-cycle duration.

Most of the mentioned OPCPA-systems are based on oxide crystals, limiting their emission wavelength range to below $5 \mu \mathrm{m}$ (see section 4.1). It is expected 630 that the recent developments of high energy picosecond lasers emitting at 2 $\mu \mathrm{m}$ wavelength [97, 99, 98, 211] will enable non-oxide crystal-based OPCPAsystems, covering the entire mid-IR spectral range.

\section{Mid-IR nonlinear spectral broadening and nonlinear compression}

In this chapter we will focus on nonlinear techniques used to modulate the spectral and temporal shape of high-energy mid-IR pulses.

The maximum possible spectral bandwidth and the related transform limited pulse duration of output pulses generated by a mid-IR radiation sources (see chap. 2.-4.) is limited by various constrains like the emission spectrum of laser gain materials or the acceptance bandwidth in the case of parametric processes. To overcome these limitations various methods have been developed exploiting mix of nonlinear light-matter interactions like self-phase modulation, selfsteepening, four-wave-mixing and plasma blue-shifting. These effects strongly reshape the spatio-temporal structure of the electric field, allowing the generation of multi-octave spanning supercontinua and temporal pulse compression 645 down to the sub-2-optical-cycle regime.

First we will focus on the spectral broadening of mid-IR pulses in waveguide fiber-structures. The second part will discuss temporal compression of the spectral broadened mid-IR pulses by dispersion control or post-compensation of the added chirp during the spectral broadening process.

\subsection{Nonlinear spectral broadening in the mid-IR}

Broadening of mid-IR pulses to octave-spanning spectra has been demonstrated in a variety of waveguide geometries. An often studied group of materials is the chalcogenide family - relying on Sulphur, Selenium or Tellurium covalently bonded to Germanium, Antimony, Gallium, Silicon or Phosphate. They exhibit nonlinear coefficients 2 to 4 orders of magnitude higher than fused silica 212 and very broad spectral transmission. Sulphides typically transmit up to $11 \mu \mathrm{m}$ wavelength, selenides transmit up to $15 \mu \mathrm{m}$ wavelength and tellurides transmit beyond $20 \mu \mathrm{m}$ wavelength [212]. Our group, in particular, recently reported spectral broadening spanning from 1.6 to $5.9 \mu \mathrm{m}$ at $-20 \mathrm{~dB}$ in an $A s_{2} S_{3}$ step660 index fiber driven by a $3.1 \mu \mathrm{m}$ laser [213. By using a longer driving wavelength at $4 \mu \mathrm{m}$ in a bulk chalcogenide glass a spectrum spanning from $2.5 \mu \mathrm{m}$ to 7.5 $\mu \mathrm{m}$ could be demonstrated [214]. Simulations indicate that pumping an As-Se chalcogenide fiber at $4.5 \mu \mathrm{m}$ could generate a spectrum from $3 \mu \mathrm{m}$ to $12 \mu \mathrm{m}$ wavelength 215. Perhaps the currently most interesting material is the fluoride compound $\mathrm{ZrF}_{4}-\mathrm{BaF}_{2}-\mathrm{LaF}_{3}-\mathrm{AlF}_{3}-\mathrm{NaF}$ better known as ZBLAN [216]. This 
material shows transparency ranging from $300 \mathrm{~nm}$ to $7 \mu \mathrm{m}$ wavelength, it can be drawn into fibers [217, and exhibits a nonlinearity similar to fused silica. ZBLAN fibers have been extensively used to efficiently produce supercontinua extending into the mid-IR spectral range by pumping above the zero-dispersion wavelength of these fibers $(1.65-1.9 \mu \mathrm{m})$ [218]. Mid-IR supercontinuum sources have been developing towards high output power [219, for example, while initial supercontinuum generation spanning up to $4.5 \mu \mathrm{m}$ [220] had been reported, the more recent use of Tm fiber lasers as pump allowed scaling up these broad sources to the watt level [221, 222], up to values as high as $62.1 \mathrm{~W}$ [223].

\subsection{Nonlinear self-compression techniques in the mid-IR}

Nonlinear pulse compression techniques of high-energy pulses rely typically on spectral broadening during self-guided filamentation in gases or bulk materials 224 and the post-compensation of the added chirp. This compression technique has been introduced approximately 10 years ago in the near-IR at the ubiquitous wavelength of $800 \mathrm{~nm}$ 225] and led to routine generation of high energy few-cycle pulses [226, 227, 228]. In this case the spectrum is typically broadened due to filamentary propagation in gases and post-compressed by a chirped mirror based compressor [226]. Optical pulses as short as 5 femtoseconds with as much as $10 \mathrm{~mJ}$ have been reported from such systems at $800 \mathrm{~nm}$ wavelength [227. By applying two compression setups in series a further pulse duration decrease to 3.8 femtoseconds could be shown 228. Supercontinuum generation in bulk GaAs and dispersion compensation in a combination of $\mathrm{BaF} 2$, $\mathrm{CaF} 2$ and $\mathrm{MgF} 2$ plates, achieved temporal pulse compression at 6 um center wavelength down to 29 femtoseconds (sub-2-optical-cycles) [229].

Another elegant alternative is self-compression 224. In this case the spectral broadening and spectral phase control are handled simultaneously, as opposed to traditional nonlinear pulse compression techniques. Since self-compression of mid-IR pulses relies on the delicate interplay of various nonlinear effects in the anomalous dispersion regime, the compression is strongly dependent of the input

695 pulse characteristics and the propagation material. For a driving wavelength of $2 \mu \mathrm{m}$ Hauri et al. have shown self-compression due to filamentation in Xenon gas from 55 femtoseconds down to 18 femtoseconds (sub-3-cycle) pulse duration [230. The possibility of transferring this compression technique in gases to the mid-IR was numerically investigated and revealed potential for quasi-single cycle pulse generation 231 but nevertheless until now just supercontinuum generation over three octaves driven at $3.9 \mu \mathrm{m}$ wavelength in Argon gas could be demonstrated [204].

A different alternative in the mid-IR is self-compression in bulk materials. Hemmer et al. pursued and pioneered an alternative approach that led to the successful self-compression of multi-cycle mid-IR optical pulses down to the fewcycle regime with high conversion efficiencies of $65 \%$ 232, 233]. The approach relies on using filamentary propagation in a thin YAG plate to generate a high spectral energy density supercontinuum spanning over 3.3 octaves from 450 $\mathrm{nm}$ to $4.5 \mu \mathrm{m}$ 232. Further, due to thorough modelling of the filamentary propagation in the crystal one can tailor the beam and crystal parameters to 
interrupt the filamentary propagation when the propagating pulse experience spatio-temporal collapse. Guided by these numerical simulations 2-fold pulse self-compression at $3.1 \mu \mathrm{m}$ wavelength from 6 -cycle duration down to sub-3-cycle could be demonstrated (see Fig. 13) [233. The scalability of the process was 715 demonstrated within the range of $3 \mu \mathrm{J}$ to $15 \mu \mathrm{J}$ input pulse energy and recently Pugzlys et al. reported further scalability to the mJ level of this process making it highly promising as a compact and efficient solid-state nonlinear compressor 234.
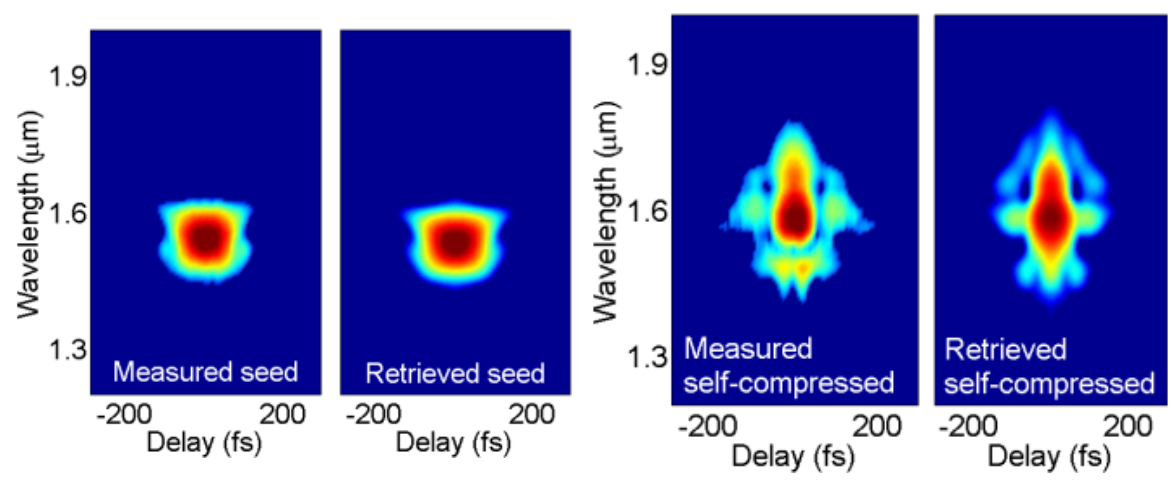

Figure 13: Measured and retrieved SHG-FROG traces of the seed (left) and self-compressed (right) pulses on logarithmic color scale.

\section{Conclusions}

Mid-IR laser sources are of great scientific and industrial importance and span applications over several scientific and technical fields. Mid-IR spectroscopy serves multiple purposes across scientific and medical applications, since gas molecules rovibrational states cover 3-15 $\mu \mathrm{m}$. Consequentially there is a need for broadband (ideally several octaves) or easily tuneable systems. High energy few-cycle and CEP stable mid-IR pulses are expected to enable the second revolution in strong field physics since attosecond-duration X-ray pulses can be generated.

These existing applications are enabled by a multitude of old and new technologies. Lead-salt and quantum cascade lasers can operate through the whole mid-IR region but can not supply ultra short pulse duration high power systems. Thulium and Holmium lasers allow for solid-state laser operating near $2 \mu \mathrm{m}$ which are suitable to pump mid-IR OPCPA systems. Namely, for up to $5 \mu \mathrm{m}$ sources Chromium or Iron-doped media lasers can be used, and DFG and OPA can be used to both extend and amplify laser pulses throughout the mid-IR. Supercontinuum generation can be achieved through SPM, compactly generating octave spanning spectra, although at the cost of efficiency. 
The mid-IR laser field has seen an accelerated development and interest in the past few years, and will continue to do so, as commercial laser and adequate pump systems are becoming available. The progresses in varying laser architectures in mid-IR lasers (namely fiber and thin-disk) have and will allow mid-IR systems to both scale in power and become more compact, thus enabling a revolution in mid-IR science. We are witnessing an exciting time in this field.

\section{Acknowledgments}

We acknowledge support from MINISTERIO DE ECONOMIA Y COMPETITIVIDAD through Plan Nacional (FIS2011-30465-C02-01), the Catalan Agencia de Gestio d'Ajuts Universitaris i de Recerca (AGAUR) with SGR 20142016, Fundacio Cellex Barcelona and LASERLAB-EUROPE grant agreement 284464 .

\section{References}

[1] F. K. Tittel, D. Richter, A. Fried, Mid-Infrared Laser Applications in Spectroscopy, in: D. I. T. Sorokina, D. K. L. Vodopyanov (Eds.), SolidState Mid-Infrared Laser Sources, no. 89 in Topics in Applied Physics, Springer Berlin Heidelberg, 2003, pp. 458-529. URL http://link.springer.com/chapter/10.1007/3-540-36491-9_ 11

[2] J. Dura, N. Camus, A. Thai, A. Britz, M. Hemmer, M. Baudisch, A. Senftleben, C. D. Schröter, J. Ullrich, R. Moshammer, J. Biegert, Ionization with low-frequency fields in the tunneling regime, Scientific Reports 3. doi:10.1038/srep02675.

URL http://www.nature.com/srep/2013/130917/srep02675/full/ srep02675.html

[3] M. G. Pullen, J. Dura, B. Wolter, M. Baudisch, M. Hemmer, N. Camus, A. Senftleben, C. D. Schroeter, R. Moshammer, J. Ullrich, J. Biegert,
Kinematically complete measurements of strong field ionization with midIR pulses, Journal of Physics B: Atomic, Molecular and Optical Physics 47 (20) (2014) 204010. doi:10.1088/0953-4075/47/20/204010. URL http://iopscience.iop.org/0953-4075/47/20/204010

[4] B. Wolter, C. Lemell, M. Baudisch, M. G. Pullen, X.-M. Tong, M. Hemmer, A. Senftleben, C. D. Schröter, J. Ullrich, R. Moshammer, J. Biegert, J. Burgdörfer, Formation of very-low-energy states crossing the ionization threshold of argon atoms in strong mid-infrared fields, Physical Review A 90 (6) (2014) 063424. doi:10.1103/PhysRevA.90.063424. URL http://link.aps.org/doi/10.1103/PhysRevA.90.063424 
[5] C. I. Blaga, J. Xu, A. D. DiChiara, E. Sistrunk, K. Zhang, P. Agostini,

[8] I. Mingareev, F. Weirauch, A. Olowinsky, L. Shah, P. Kadwani, M. Richardson, Welding of polymers using a $2 \mu \mathrm{m}$ thulium

[10] J. Faist, F. Capasso, D. L. Sivco, C. Sirtori, A. L. Hutchinson, A. Y. Cho, Quantum Cascade Laser, Science 264 (5158) (1994) 553-556. doi: 10.1126/science.264.5158.553

URL http://www. sciencemag. org/content/264/5158/553

[11] Y. Bonetti, J. Faist, Quantum cascade lasers: Entering the mid-infrared Nature Photonics 3 (1) (2009) 32-34. doi:10.1038/nphoton.2008.268.

810

http://www .nature.com/nphoton/journal/v3/n1/full/ nphoton.2008.268.html

[12] Y. Bai, N. Bandyopadhyay, S. Tsao, S. Slivken, M. Razeghi, Room temperature quantum cascade lasers with $27 \%$ wall plug efficiency, Ap- 

3586773 .

[13] P. Q. Liu, A. J. Hoffman, M. D. Escarra, K. J. Franz, J. B. Khurgin, " Y. Dikmelik, X. Wang, J.-Y. Fan, C. F. Gmachl, Highly powerefficient quantum cascade lasers, Nature Photonics 4 (2) (2010) 95-98. doi:10.1038/nphoton.2009.262. URL http://www.nature.com/nphoton/journal/v4/n2/full/ nphoton.2009.262.html

[14] Y. Yao, A. J. Hoffman, C. F. Gmachl, Mid-infrared quantum cascade lasers, Nature Photonics 6 (7) (2012) 432-439. doi:10.1038/nphoton.2012.143

URL http://www.nature.com/nphoton/journal/v6/n7/full/ nphoton.2012.143.html

[15] P. Patimisco, V. Spagnolo, M. Vitiello, A. Tredicucci, G. Scamarcio, C. Bledt, J. Harrington, Coupling external cavity mid-IR quantum cascade lasers with low loss hollow metallic/dielectric waveguides, Applied Physics B 108 (2) (2012) 255-260. doi:10.1007/s00340-012-4891-2. URL http://dx.doi .org/10.1007/s00340-012-4891-2

[16] O. Cathabard, R. Teissier, J. Devenson, J. C. Moreno, A. N. Baranov, Quantum cascade lasers emitting near $2.6 \mu \mathrm{m}$, Applied Physics Letters 96 (14) (2010) 141110. doi:10.1063/1.3385778. URL http://scitation.aip.org/content/aip/journal/apl/96/14/ $10.1063 / 1.3385778$

[17] A. Lyakh, R. Maulini, A. Tsekoun, R. Go, C. Pflügl, L. Diehl, Q. J. Wang, F. Capasso, C. K. N. Patel, 3 W continuous-wave room temperature single-facet emission from quantum cascade lasers based on nonresonant extraction design approach, Applied Physics Letters 95 (14) (2009) 141113. doi:10.1063/1.3238263.

n URL http://scitation.aip.org/content/aip/journal/apl/95/14/ $10.1063 / 1.3238263$

[18] D. G. Revin, J. W. Cockburn, M. J. Steer, R. J. Airey, M. Hopkinson, A. B. Krysa, L. R. Wilson, S. Menzel, InGaAs / AlAsSb / InP quantum cascade lasers operating at wavelengths close to $3 \mu \mathrm{m}$, Applied Physics Letters 90 (2) (2007) 021108. doi:10.1063/1.2431035 URL http://scitation.aip.org/content/aip/journal/apl/90/2/ $10.1063 / 1.2431035$

[19] J. Maysonnave, K. Maussang, J. R. Freeman, N. Jukam, J. Madéo, P. Cavalié, R. Rungsawang, S. P. Khanna, E. H. Linfield, A. G. Davies,

I H. E. Beere, D. A. Ritchie, S. S. Dhillon, J. Tignon, Mode-locking of a terahertz laser by direct phase synchronization, Opt. Express 20 (19) (2012) 20855-20862. doi:10.1364/0E.20.020855. 


1) URL he-20-19-20855 http://www.opticsexpress.org/abstract.cfm?URI=

[20] R. Paiella, F. Capasso, C. Gmachl, H. Y. Hwang, D. L. Sivco, A. L. n Hutchinson, A. Y. Cho, H. C. Liu, Monolithic active mode locking of quantum cascade lasers, Applied Physics Letters 77 (2) (2000) 169-171. doi:http://dx.doi.org/10.1063/1.126913.

1 URL http://scitation.aip.org/content/aip/journal/apl/77/2/ $10.1063 / 1.126913$

[21] C. Y. Wang, L. Diehl, A. Gordon, C. Jirauschek, F. X. Kärtner, A. Belyanin, D. Bour, S. Corzine, G. Höfler, M. Troccoli, J. Faist, F. Ca1 $\quad$ passo, Coherent instabilities in a semiconductor laser with fast gain recovery, Phys. Rev. A 75 (3) (2007) 031802. doi:10.1103/PhysRevA.75. 031802 .

URL http://link. aps .org/doi/10.1103/PhysRevA.75.031802

${ }_{870}^{\text {¿ }}[22]$ A. Hugi, G. Villares, S. Blaser, H. C. Liu, J. Faist, Mid-infrared frequency comb based on a quantum cascade laser, Nature 492 (7428) (2012) 229233. doi:10.1038/nature11620. URL http://dx.doi .org/10.1038/nature11620

[23] G. Villares, A. Hugi, S. Blaser, J. Faist, Dual-comb spectroscopy based on quantum-cascade-laser frequency combs, Nat Commun 5.

URL http://dx.doi.org/10.1038/ncomms6192

[24] C. Y. Wang, L. Kuznetsova, V. M. Gkortsas, L. Diehl, F. X. Kärtner, M. A. Belkin, A. Belyanin, X. Li, D. Ham, H. Schneider, P. Grant, C. Y.

口. Song, S. Haffouz, Z. R. Wasilewski, H. Liu, F. Capasso, Mode-locked ${ }_{880} \quad$ pulses from mid-infrared Quantum Cascade Lasers, Optics Express 17 (15) (2009) 12929-12943. doi:10.1364/OE.17.012929.

I URL http://www.opticsexpress.org/abstract.cfm?URI= oe-17-15-12929

[25] A. K. Wojcik, P. Malara, R. Blanchard, T. S. Mansuripur, F. Capasso, 885 A. Belyanin, Generation of picosecond pulses and frequency combs in actively mode locked external ring cavity quantum cascade lasers, Applied Physics Letters 103 (23). doi:http://dx.doi.org/10.1063/1.4838275.

a URL http://scitation.aip.org/content/aip/journal/apl/103/23/ $10.1063 / 1.4838275$

${ }_{890}^{\text {口 }}[26]$ U. P. Schießl, J. Rohr, 60 C lead salt laser emission near $5 \mu \mathrm{m}$ wavelength, Infrared Physics \& Technology 40 (4) (1999) 325-328. doi:10.1016/S1350-4495(99)00028-6.

a URL http://www.sciencedirect.com/science/article/pii/ S1350449599000286

${ }_{895}^{2}$ [27] P. C. Findlay, C. R. Pidgeon, R. Kotitschke, A. Hollingworth, B. N. Murdin, C. J. G. M. Langerak, A. F. G. van der Meer, C. M. Ciesla, J. Oswald, 
[30] A. Khiar, V. Volobuev, M. Witzan, A. Hochreiner, M. Eibelhuber, 915 ㅁ G. Springholz, In-well pumped mid-infrared $\mathrm{PbTe} / \mathrm{CdTe}$ quantum well vertical external cavity surface emitting lasers, Applied Physics Letters 104 (23) (2014) 231105. doi:10.1063/1.4882081.

口

[33] T. Schwarzl, W. Heiss, G. Springholz, M. Aigle, H. Pascher, $6 \mu \mathrm{m}$ vertical cavity surface emitting laser based on IV-VI semiconductor compounds, Electronics Letters 36 (4) (2000) 322-324. doi:10.1049/el:20000260.

\footnotetext{
[ [34] F. Zhao, H. Wu, L. Jayasinghe, Z. Shi, Above-room-temperature 935 a optically pumped $4.12 \mu \mathrm{m}$ midinfrared vertical-cavity surfaceemitting lasers, Applied Physics Letters 80 (7) (2002) 1129-1131. doi:http://dx.doi.org/10.1063/1.1449540.
} 

$10.1063 / 1.1449540$

940

[37] J. Furst, T. Schwarzl, M. Boberl, H. Pascher, G. Springholz, W. Heiss, Vertical-cavity surface-emitting lasers in the $8-\mu \mathrm{m}$ midinfrared spectral range with continuous-wave and pulsed emission, Quantum Electronics, IEEE Journal of 40 (8) (2004) 966-969. doi:10.1109/JQE.2004.831634.

[38] S. Bowman, J. Ganem, B. Feldman, A. Kueny, Infrared laser characteristics of praseodymium-doped lanthanum trichloride, IEEE Journal of Quantum Electronics 30 (12) (1994) 2925-2928. doi:10.1109/3.362716.

[39] S. Bowman, L. Shaw, B. Feldman, J. Ganem, A $7 \mu$ m praseodymiumbased solid-state laser, IEEE Journal of Quantum Electronics 32 (4) (1996) 646-649. doi:10.1109/3.488838.

[40] L. Sojka, Z. Tang, H. Zhu, E. Beres-Pawlik, D. Furniss, A. B. Seddon, - T. M. Benson, S. Sujecki, Study of mid-infrared laser action in chalco965 genide rare earth doped glass with Dy3+, Pr3+and Tb3+, Optical Materials Express 2 (11) (2012) 1632-1640. doi:10.1364/OME.2.001632.

a URL http://www.opticsinfobase.org/ome/abstract.cfm?URI= ome-2-11-1632

[ [41] U. Hömmerich, E. Brown, P. Amedzake, S. B. Trivedi, J. M. Zavada, Midinfrared $(4.6 \mu \mathrm{m})$ emission properties of Pr3+ doped KPb2br5, Journal of Applied Physics 100 (11) (2006) 113507. doi:10.1063/1.2399888.

1. URL http://scitation.aip.org/content/aip/journal/jap/100/11/ $10.1063 / 1.2399888$

[42] A. G. Okhrimchuk, L. N. Butvina, E. M. Dianov, I. A. Shestakova, N. V. 975 Lichkova, V. N. Zagorodnev, A. V. Shestakov, Optical spectroscopy of 1. the RbPb2cl5:Dy3+ laser crystal and oscillation at $5.5 \mu \mathrm{m}$ at room temperature, Journal of the Optical Society of America B 24 (10) (2007) 
2690-2695. doi:10.1364/JOSAB.24.002690.

URL http://josab.osa.org/abstract.cfm?URI=josab-24-10-2690

980

985

995

1005

1010

[49] R. Peters, C. Kränkel, S. T. Fredrich-Thornton, K. Beil, K. Petermann, G. Huber, O. H. Heckl, C. R. E. Baer, C. J. Saraceno, T. Südmeyer, 1015 U. Keller, Thermal analysis and efficient high power continuous-wave and mode-locked thin disk laser operation of Yb-doped sesquioxides, Applied Physics B 102 (3) (2011) 509-514. doi:10.1007/s00340-011-4428-0.

n- URL s00340-011-4428-0 http://link.springer.com/article/10.1007/ 
1020

[57] L. J. Johnson, G. D. Boyd, K. Nassau, Optical maser characteristics of Ho3+ in CaWO4, Proc IAE (1962) 87-90.

[58] E. P. Chicklis, C. S. Naiman, R. C. Folweiler, D. R. Gabbe, H. P.

Jenssen, A. Linz, High-Efficiency Room Temperature $2.06 \mu \mathrm{m}$ Laser

U. Griebner, V. Petrov, K. Petermann, V. Peters, Passively modelocked Yb:Lu2o3 laser, Optics Express 12 (14) (2004) 3125-3130. doi:10.1364/OPEX.12.003125.

URL http://www.opticsexpress.org/abstract.cfm?URI= oe-12-14-3125

[51] W. Kim, C. Baker, C. Florea, J. Frantz, G. Villalobos, B. Shaw, S. Bowman, S. O'Connor, B. Sadowski, M. Hunt, I. Aggalwar, J. Sanghera, Doped sesquioxide ceramic for eye-safe solid state laser materials, Vol. 8599, 2013, pp. 85990J-85990J-6. doi:10.1117/12.2004524.

URL http://dx.doi.org/10.1117/12.2004524

[52] P. Koopmann, S. Lamrini, K. Scholle, C. Krankel, P. Fuhrberg, G. Huber, Holmium-Doped Lutetia: A Novel Diode Pumped Laser at $2124 \mathrm{~nm}$, in: Lasers, Sources, and Related Photonic Devices, OSA Technical Digest (CD), Optical Society of America, 2012, p. IW5D.4. doi:10.1364/AIOM.2012.IW5D.4

URL http://www.opticsinfobase.org/abstract.cfm?URI=
AIOM-2012-IW5D . 4

[53] G. A. Newburgh, A. Word-Daniels, A. Michael, L. D. Merkle, A. Ikesue, M. Dubinskii, Resonantly diode-pumped Ho3+:Y2o3 ceramic $2.1 \mu \mathrm{m}$ laser, Optics Express 19 (4) (2011) 3604-3611. doi:10.1364/OE.19.003604. URL http://www.opticsexpress.org/abstract.cfm?URI= oe-19-4-3604

[54] V. Fedorov, S. Mirov, A. Gallian, D. Badikov, M. Frolov, Y. Korostelin, V. Kozlovsky, A. Landman, Y. Podmar'kov, V. Akimov, A. Voronov, 3.77$5.05 \mu \mathrm{m}$ tunable solid-state lasers based on Fe2+-doped ZnSe crystals operating at low and room temperatures, Quantum Electronics, IEEE Journal of 42 (9) (2006) 907-917. doi:10.1109/JQE.2006.880119.

[55] H. Jelínková, P. Koranda, M. E. Doroshenko, J. Šulc, M. Jelínek, M. Cech, T. T. Basiev, V. V. Badikov, D. V. Badikov, Room-temperature lasing, gain-switched bulk, tunable Fe:ZnSe laser, Vol. 7721, 2010, pp. 772111772111-6. doi:10.1117/12.854304. URL http://dx.doi .org/10.1117/12.854304

of $4.3 \mu \mathrm{m}$ room temperature Fe:ZnSe laser Optics Letters $36(1)(2011)$ 94-96. doi:10.1364/0L.36.000094 URL http://ol.osa.org/abstract.cfm?URI=ol-36-1-94 Using Sensitized Ho3+:YLF, Applied Physics Letters 19 (4) (1971) 
119-121. doi:http://dx.doi.org/10.1063/1.1653837.

1. URL http://scitation.aip.org/content/aip/journal/apl/19/4/ $10.1063 / 1.1653837$

[59] G. A. Slack, F. S. Ham, R. M. Chrenko, Optical Absorption of Tetrahedral Fe2+ (3d6) in Cubic ZnS, CdTe, and MgAl2o4, Phys. Rev. 152 (1) (1966) 376-402. doi:10.1103/PhysRev.152.376. URL http://link.aps .org/doi/10.1103/PhysRev.152.376

[60] G. A. Slack, B. M. O'Meara, Infrared Luminescence of $\mathrm{Fe} 2+$ in ZnS, Physical Review 163 (1967) 335-340. doi:10.1103/PhysRev.163.335.

${ }_{1070}$ [61] H. Nelkowski, G. Grebe, IR-luminescence of ZnS:Cr, Journal of Luminescence 1 (0) (1970) 88 - 93. doi:http://dx.doi.org/10.1016/ 0022-2313(70)90025-6.

口 URL http://www.sciencedirect.com/science/article/pii/ 0022231370900256

1075 [62] J. T. Vallin, G. A. Slack, S. Roberts, A. E. Hughes, Infrared Absorption in Some II-VI Compounds Doped with Cr, Phys. Rev. B 2 (11) (1970) 4313-4333. doi:10.1103/PhysRevB.2.4313. URL http://link. aps . org/doi/10.1103/PhysRevB.2.4313

[63] G. Grebe, G. Roussos, H.-J. Schulz, Infrared luminescence of ZnSe:Cr crystals, Journal of Luminescence 12 (0) (1976) 701 - 705, proceeding of the 1975 International Conference on Luminescence. doi:http://dx.doi.org/10.1016/0022-2313(76)90164-2.

a URL http://www.sciencedirect.com/science/article/pii/ 0022231376901642

1085 [64] M. Godlewski, M. Kaminska, The chromium impurity photogeneration transitions in ZnS, ZnSe and ZnTe, Journal of Physics C: Solid State Physics 13 (35) (1980) 6537. doi:10.1088/0022-3719/13/35/013. URL http://iopscience.iop.org/0022-3719/13/35/013

[ [65] G. Roussos, H.-J. Schulz, M. Thiede, Luminescence and related optical

1090 properties of iron ions in II-VI compounds, Journal of Luminescence
31-32, Part 1 (0) (1984) 409-411. doi:10.1016/0022-2313(84)90310-7.
URL $\quad$ http://www.sciencedirect.com/science/article/pii/

$1090 \begin{aligned} & \text { properties of iron ions in II-VI compounds, Journal of Luminescence } \\ & \text { 31-32, Part 1 (0) (1984) 409-411. doi:10.1016/0022-2313(84)90310-7. } \\ & \text { URL } \quad \text { http://www.sciencedirect.com/science/article/pii/ }\end{aligned}$ 0022231384903107

[66] J. M. Baranowski, J. W. Allen, G. L. Pearson, Crystal-Field Spectra of ${ }_{1095}$ dn Impurities in II-VI and III-V Compound Semiconductors, Phys. Rev. 160 (3) (1967) 627-632. doi:10.1103/PhysRev.160.627. URL http://link. aps .org/doi/10.1103/PhysRev.160.627

[67] G. A. Slack, S. Roberts, F. S. Ham, Far-Infrared Optical Absorption of $\mathrm{Fe} 2+$ in ZnS, Physical Review 155 (1967) 170-177. doi:10.1103/ $1100 \quad$ PhysRev.155.170. 
[68] L. D. DeLoach, R. H. Page, G. D. Wilke, S. A. Payne, W. F. Krupke, Transition metal-doped zinc chalcogenides: spectroscopy and laser demonstration of a new class of gain media, Quantum Electronics, IEEE Journal of 32 (6) (1996) 885-895.

1105 URL http://ieeexplore.iee.org/xpls/abs_all.jsp?arnumber= 502365

[69] V. A. Akimov, V. I. Kozlovskii, Y. V. Korostelin, A. I. Landman, Y. P. Podmar'kov, Y. K. Skasyrsky, M. P. Frolov, Efficient cw lasing in a Cr2+:CdSe crystal, Quantum Electronics 37 (11) (2007) 991-992. doi:10.1070/QE2007v037n11ABEH013715.

[70] E. Sorokin, D. Klimentov, M. P. Frolov, Y. V. Korostelin, V. I. Kozlovsky, Y. P. Podmar'kov, Y. K. Skasyrsky, I. T. Sorokina, Continuous-wave 1115 broadly tunable high-power Cr:CdS laser, Applied Physics B (2014) 1-6doi:10.1007/s00340-014-5921-z.

1 URL http://link.springer.com/article/10.1007/ s00340-014-5921-z

[71] V. Kisel, N. Tolstik, V. Shcherbitsky, N. Kuleshov, V. Konstantinov, ${ }_{1120}$ L. Postnova, V. Levchenko, E. Sorokin, I. Sorokina, Growth and spectroscopic characterization of Cr:ZnTe laser crystals, in: 2005 Conference on Lasers and Electro-Optics Europe, 2005. CLEO/Europe, 2005, pp. 81-. doi:10.1109/CLEOE.2005.1567870

[72] S. D. Jackson, Cross relaxation and energy transfer upconversion processes relevant to the functioning of $2 \mu \mathrm{m}$ Tm3+-doped silica fibre lasers, Optics Communications 230 (1-3) (2004) 197-203. doi:10.1016/j.optcom.2003.11.045.

II URL http://www.sciencedirect.com/science/article/pii/ S0030401803022612

${ }_{1130}[73]$ D. Y. Shen, J. K. Sahu, W. A. Clarkson, High-power widely tunable Tm:fibre lasers pumped by an Er,Yb co-doped fibre laser at $1.6 \mu \mathrm{m}$, Optics Express 14 (13) (2006) 6084-6090. doi:10.1364/OE.14.006084.

I URL http://www.opticsexpress.org/abstract.cfm?URI= oe-14-13-6084

1135 [74] D. Creeden, B. R. Johnson, S. D. Setzler, E. P. Chicklis, Resonantly pumped Tm-doped fiber laser with $>90 \%$ slope efficiency, Optics Letters 39 (3) (2014) 470-473. doi:10.1364/0L.39.000470 URL http://ol .osa.org/abstract.cfm?URI=ol-39-3-470

[75] P. Moulton, G. Rines, E. Slobodtchikov, K. Wall, G. Frith, B. Samson, A. Carter, Tm-Doped Fiber Lasers: Fundamentals and Power Scaling, IEEE Journal of Selected Topics in Quantum Electronics 15 (1) (2009) 85-92. doi:10.1109/JSTQE.2008.2010719. 
[76] T. S. McComb, R. A. Sims, C. C. C. Willis, P. Kadwani, V. Sudesh, L. Shah, M. Richardson, High-power widely tunable thulium fiber lasers, Applied Optics 49 (32) (2010) 6236-6242. doi:10.1364/A0.49.006236. URL http: //ao.osa.org/abstract .cfm?URI=ao-49-32-6236

[77] T. S. McComb, L. Shah, R. A. Sims, C. C. C. Willis, P. Kadwani, V. Sudesh, M. Richardson, Atmospheric propagation testing with a high power, tunable thulium fiber laser system, Vol. 7578, 2010, pp. 75781G75781G-7. doi:10.1117/12.842378 URL http://dx .doi .org/10.1117/12.842378

[78] Q. Fang, W. Shi, K. Kieu, E. Petersen, A. Chavez-Pirson, N. Peyghambarian, High power and high energy monolithic single frequency $2 \mu \mathrm{m}$ nanosecond pulsed fiber laser by using large core Tm-doped germanate fibers: experiment and modeling, Optics Express 20 (15) (2012) 1641016420. doi:10.1364/OE.20.016410.

a URL http://wWw.opticsexpress.org/abstract.cfm?URI= oe-20-15-16410

[79] P. Kadwani, N. Modsching, R. A. Sims, L. Leick, J. Broeng, L. Shah, M. Richardson, Q-switched thulium-doped photonic crystal fiber laser, Optics Letters 37 (10) (2012) 1664-1666. doi:10.1364/0L.37.001664. URL http://ol .osa.org/abstract.cfm?URI=ol-37-10-1664

[80] M. Gebhardt, C. Gaida, P. Kadwani, A. Sincore, N. Gehlich, C. Jeon, L. Shah, M. Richardson, High peak-power mid-infrared ZnGeP2 optical parametric oscillator pumped by a Tm:fiber master oscillator power amplifier system, Optics Letters 39 (5) (2014) 1212-1215. doi:10.1364/OL. 39.001212. URL http://ol .osa. org/abstract. cfm?URI=ol-39-5-1212

[81] J. B. Gruber, M. E. Hills, R. M. Macfarlane, C. A. Morrison, G. A. Turner, $1170 \quad$ G. J. Quarles, G. J. Kintz, L. Esterowitz, Spectra and energy levels of a. Tm3+:Y3al5o12, Phys. Rev. B 40 (14) (1989) 9464-9478. doi:10.1103/ PhysRevB.40.9464. URL http://link. aps .org/doi/10.1103/PhysRevB.40.9464

[82] S. Jackson, The spectroscopic and energy transfer characteristics of the rare earth ions used for silicate glass fibre lasers operating in the shortwave infrared, Laser \& Photonics Reviews 3 (5) (2009) 466-482. doi:10.1002/ 1por.200810058. URL http://dx.doi.org/10.1002/lpor.200810058

[83] M. A. Solodyankin, E. D. Obraztsova, A. S. Lobach, A. I. Chernov, A. V. $1180 \quad$ Tausenev, V. I. Konov, E. M. Dianov, Mode-locked $1.93 \mu \mathrm{m}$ thulium fiber laser with a carbon nanotube absorber, Opt. Lett. 33 (12) (2008) 13361338. doi:10.1364/OL.33.001336.

URL http://ol.osa.org/abstract .cfm?URI=0l-33-12-1336 
[84] R. A. Sims, P. Kadwani, A. S. L. Shah, M. Richardson, $1 \mu \mathrm{J}$, sub-500 fs chirped pulse amplification in a Tm-doped fiber system, Opt. Lett. 38 (2) (2013) 121-123. doi:10.1364/0L.38.000121. URL http://ol .osa.org/abstract.cfm?URI=ol-38-2-121

[ [85] R. Sims, P. Kadwani, L. Shah, M. Richardson, All Thulium Fiber CPA System with 107 fs Pulse Duration and $42 \mathrm{~nm}$ Bandwidth, in: Advances in Optical Materials, Optical Society of America, 2011, p. ATuD4. doi:10.1364/ASSP.2011.ATuD4. URL ASSP-2011-ATuD4 http://www.opticsinfobase.org/abstract.cfm?URI=

[86] S. Agger, J. Povlsen, P. Varming, Single-frequency thulium-doped distributed-feedbackfiber laser, Optics Letters 29 (13) (2004) 1503-1505. doi:10.1364/0L.29.001503.

URL http://ol.osa.org/abstract.cfm?URI=ol-29-13-1503

[87] N. Barnes, E. D. Filer, C. A. Morrison, C. Lee, Ho:Tm lasers. I. Theoretical, Quantum Electronics, IEEE Journal of 32 (1) (1996) 92-103. 1200 doi:10.1109/3.481924.

[88] B. M. Walsh, G. W. Grew, N. P. Barnes, Energy levels and intensity parameters of Ho 3+ ions in GdLiF 4, YLiF 4 and LuLiF 4, Journal of Physics: Condensed Matter 17 (48) (2005) 7643. URL http://stacks . iop. org/0953-8984/17/i=48/a=016

[89] B. M. Walsh, N. P. Barnes, M. Petros, J. Yu, U. N. Singh, Spectroscopy and modeling of solid state lanthanide lasers: Application to trivalent Tm3+ and Ho3+ in YLiF4 and LuLiF4, Journal of Applied Physics 95 (7) (2004) 3255-3271. doi:http://dx.doi.org/10.1063/1.1649808.

a URL http://scitation.aip.org/content/aip/journal/jap/95/7/ $1210 \quad 10.1063 / 1.1649808$

[90] N. P. Barnes, B. M. Walsh, E. D. Filer, Ho:Ho upconversion: applications 1. to Ho lasers, J. Opt. Soc. Am. B 20 (6) (2003) 1212-1219. doi:10.1364/ JOSAB. 20.001212. URL http://josab.osa.org/abstract.cfm?URI=josab-20-6-1212

1215 [91] C. Lee, G. Han, N. Barnes, Ho:Tm lasers. II. Experiments, Quantum a Electronics, IEEE Journal of 32 (1) (1996) 104-111. doi:10.1109/3. 481925 .

[92] V. Sudesh, K. Asai, Spectroscopic and diode-pumped-laser properties of Tm, Ho:YLF; Tm, Ho:LuLF; and Tm, Ho:LuAG crystals: a comparative study, J. Opt. Soc. Am. B 20 (9) (2003) 1829-1837. doi:10.1364/JOSAB. 20.001829.

URL http://josab.osa.org/abstract.cfm?URI=josab-20-9-1829 
n [93] M. Eichhorn, Quasi-three-level solid-state lasers in the near and mid infrared based on trivalent rare earth ions, Applied Physics B 93 (2-3) (2008) 269-316. doi:10.1007/s00340-008-3214-0 URL http://link. springer .com/10.1007/s00340-008-3214-0

[94] W. Koen, C. Bollig, H. Strauss, M. Schellhorn, C. Jacobs, M. J. D. Esser, Compact fibre-laser-pumped Ho:YLF oscillator-amplifier system, Applied Physics B 99 (1-2) (2010) 101-106. doi:10.1007/s00340-009-3819-y. URL http://link.springer.com/10.1007/s00340-009-3819-y

[95] F. Fusari, A. A. Lagatsky, G. Jose, S. Calvez, A. Jha, M. D. Dawson, J. A. Gupta, W. Sibbett, C. T. A. Brown, Femtosecond mode-locked Tm3+ and Tm3+-Ho3+ doped $2 \mu \mathrm{m}$ glass lasers, Opt. Express 18 (21) (2010) 22090-22098. doi:10.1364/0E.18.022090.

1235 a URL http://www.opticsexpress.org/abstract.cfm?URI= oe-18-21-22090

n [96] H. Fonnum, E. Lippert, M. W. Haakestad, $550 \mathrm{~mJ}$ Q-switched cryogenic Ho:YLF oscillator pumped with a $100 \mathrm{~W}$ Tm:fiber laser, Optics Letters 38 (11) (2013) 1884-1886. doi:10.1364/0L .38.001884. URL http: //ol .osa.org/abstract . cfm?URI=ol-38-11-1884

[97] P. Malevich, G. Andriukaitis, T. Flöry, A. J. Verhoef, A. Fernández, S. Ališauskas, A. Pugžlys, A. Baltuška, L. H. Tan, C. F. Chua, P. B.

․ Phua, High energy and average power femtosecond laser for driving midinfrared optical parametric amplifiers, Opt. Lett. 38 (15) (2013) 27462749. doi:10.1364/0L .38.002746. URL http://ol.osa.org/abstract . cfm?URI=ol-38-15-2746

[98] M. Hemmer, D. Sanchez, M. Jelínek, H. Jelínková, V. Kubeček, J. Biegert, Fiber-seeded, 10-ps, 2050-nm, multi-mJ, cryogenic Ho:YLF CPA, in: CLEO: 2014, Optical Society of America, 2014, p. SM1F.3. doi:10.1364/CLEO_SI.2014.SM1F.3.

I URL http://www.opticsinfobase.org/abstract.cfm?URI=CLEO_ SI-2014-SM1F.3

[99] M. Hemmer, M. Baudisch, J. Biegert, Scaling Few-Cycle OPCPAs to the 4-8 $\mu \mathrm{m}$ Spectral Range: Prospect for $2 \mu \mathrm{m}$ PumpLasers,

1255 in: CLEO: 2013, Optical Society of America, 2013, p. CM1N.4. doi:10.1364/CLEO_SI.2013.CM1N.4.

I) URL http://www.opticsinfobase.org/abstract.cfm?URI=CLEO_ SI-2013-CM1N. 4

[100] P. Li, A. Ruehl, U. Grosse-Wortmann, I. Hartl, Sub-100 fs passively modelocked holmium-doped fiber oscillator operating at $2.06 \mu \mathrm{m}$, Opt. Lett. 39 (24) (2014) 6859-6862. doi:10.1364/OL.39.006859. URL http://ol.osa.org/abstract.cfm?URI=ol-39-24-6859 
[101] H. Hoogland, S. Wittek, W. Hänsel, S. Stark, R. Holzwarth, Fiber chirped pulse amplifier at $2.08 \mu \mathrm{m}$ emitting 383 -fs pulses at $10 \mathrm{~nJ}$ and $7 \mathrm{MHz}$, Opt. Lett. 39 (23) (2014) 6735-6738. doi:10.1364/0L.39.006735. URL http://ol.osa.org/abstract .cfm?URI=ol-39-23-6735

[102] E. Sorokin, S. Naumov, I. T. Sorokina, Ultrabroadband infrared solidstate lasers, Selected Topics in Quantum Electronics, IEEE Journal of 11 (3) (2005) 690-712.

[103] S. B. Mirov, V. V. Fedorov, D. V. Martyshkin, I. S. Moskalev, M. S. Mirov, V. P. Gapontsev, Progress in mid-IR Cr2+ and Fe2+ doped II-VI materials and lasers [Invited], Optical Materials Express 1 (5) (2011) 898-910. doi:10.1364/OME.1.000898.

[1] URL http://www.opticsinfobase.org/ome/abstract.cfm?URI= ome-1-5-898

[104] N. Tolstik, E. Sorokin, V. Kalashnikov, D. Klimentov, V. Dvoyrin, I. T. Sorokina, Supercontinuum generation in mid-IR using chalcogenide and

$1280 \quad$ germanate nonlinear fiber, Vol. 8599, 2013, pp. 85990K-85990K-6. doi: 10.1117/12.2003957.

URL http://dx.doi.org/10.1117/12.2003957

[105] E. Sorokin, I. Sorokina, Ultrashort-pulsed Kerr-lens modelocked Cr:ZnSe laser, in: Lasers and Electro-Optics 2009 and the European Quantum 1285 Electronics Conference. CLEO Europe - EQEC 2009. European Conference on, 2009, pp. 1-1. doi:10.1109/CLEOE-EQEC.2009.5196427.

[106] M. N. Cizmeciyan, H. Cankaya, A. Kurt, A. Sennaroglu, Kerr-lens modelocked femtosecond $\mathrm{Cr} 2+: \mathrm{ZnSe}$ laser at $2420 \mathrm{~nm}$, Optics Letters $34(20)$ (2009) 3056-3058. doi:10.1364/0L .34.003056. URL http://ol .osa .org/abstract . cfm?URI=ol-34-20-3056

[107] E. Sorokin, N. Tolstik, I. T. Sorokina, Femtosecond operation and self-doubling of Cr:ZnS laser, in: Nonlinear Optics, Optical Society of America, 2011, p. NThC1. doi:10.1364/NL0.2011.NThC1. URL NLO-2011-NThC1

[108] N. Tolstik, E. Sorokin, I. T. Sorokina, Kerr-lens mode-locked Cr:ZnS laser Opt. Lett. 38 (3) (2013) 299-301. doi:10.1364/0L.38.000299. URL http://ol.osa.org/abstract.cfm?URI=ol-38-3-299

[109] E. Slobodtchikov, P. Moulton, Progress in Ultrafast Cr:ZnSe Lasers, in: Lasers, Sources, and Related Photonic Devices, Optical Society of America, 2012, p. AW5A.4. doi:10.1364/ASSP. 2012.AW5A.4.

a URL http://www.opticsinfobase.org/abstract.cfm?URI= ASSP-2012-AW5A.4 
[110] K. Schepler, R. Peterson, P. Berry, J. McKay, Thermal effects in

Cr2+:ZnSe thin disk lasers, IEEE Journal of Selected Topics in Quantum Electronics 11 (3) (2005) 713-720. doi:10.1109/JSTQE.2005.850570.

URL http://ieeexplore.ieee.org/lpdocs/epic03/wrapper.htm? arnumber $=1516138$

[111] G. Renz, J. Speiser, A. Giesen, I. Sorokina, E. Sorokin, Cr:ZnSe Bulk and Cr:ZnSe Thin Disk cw Lasers, in: Lasers, Sources, and Related Photonic Devices, Optical Society of America, 2012, p. AT4A.3. doi:10.1364/ASSP. 2012.AT4A.3

1 URL http://www.opticsinfobase.org/abstract.cfm?URI= ASSP-2012-AT4A.3

[112] J. J. Adams, C. Bibeau, R. H. Page, D. M. Krol, L. H. Furu, S. A. Payne, 4.0-4.5- $\mu \mathrm{m}$ lasing of Fe:ZnSe below $180 \mathrm{~K}$, a new mid-infrared laser material, Opt. Lett. 24 (23) (1999) 1720-1722. doi:10.1364/OL. 24.001720 URL http://ol.osa.org/abstract .cfm?URI=ol-24-23-1720

[113] J. Evans, P. Berry, K. Schepler, A Passively Q -Switched, CW-Pumped Fe:ZnSe Laser, Quantum Electronics, IEEE Journal of 50 (3) (2014) 204209. doi:10.1109/JQE. 2014.2302233.

[114] Y. Song, J. Sonntag, S. B. Mirov, C. F. Gmachl, J. B. Khurgin, Midinfrared light emission from a $\mathrm{Fe} 2+: \mathrm{ZnSe}$ polycrystal using quantum cascade laser pumping, Applied Physics Letters 105 (14) (2014) 141108. doi:10.1063/1.4897546.

URL http://scitation.aip.org/content/aip/journal/apl/105/14/ $10.1063 / 1.4897546$

[115] P. A. Franken, A. E. Hill, C. W. Peters, G. Weinreich, Generation of Optical Harmonics, Phys. Rev. Lett. 7 (4) (1961) 118-119. doi:10.1103/ PhysRevLett.7.118.

URL http://link.aps.org/doi/10.1103/PhysRevLett.7.118

[116] T. H. Maiman, Stimulated Optical Radiation in Ruby, Nature 187 (4736) (1960) 493-494. doi:10.1038/187493a0.

URL http://www.nature.com/nature/journal/v187/n4736/abs/ 187493a0.html

117] M. Bass, P. A. Franken, A. E. Hill, C. W. Peters, G. Weinreich, Optical Mixing, Phys. Rev. Lett. 8 (1) (1962) 18-18. doi:10.1103/PhysRevLett. 8.18.

URL http://link.aps.org/doi/10.1103/PhysRevLett.8.18

[118] M. Bass, P. A. Franken, J. F. Ward, G. Weinreich, Optical Rectification Phys. Rev. Lett. 9 (11) (1962) 446-448. doi:10.1103/PhysRevLett.9. 446 .

URL http://link. aps .org/doi/10.1103/PhysRevLett .9.446 
1345

[119] A. W. Smith, N. Braslau, Observation of an Optical Difference Frequency, Journal of Applied Physics 34 (7) (1963) 2105-2106. doi:http://dx.doi.org/10.1063/1.1729748.

口

29748

[120] C. C. Wang, G. W. Racette, Measurement of parametric gain accompanying optical difference frequency generation, Applied Physics Letters 6 (8) (1965) 169-171. doi:http://dx.doi.org/10.1063/1.1754219.

[122] J. A. Giordmaine, R. C. Miller, Tunable Coherent Parametric Oscillation in LiNbO3 at Optical Frequencies, Phys. Rev. Lett. 14 (24) (1965) 973976. doi:10.1103/PhysRevLett.14.973. URL http://link.aps.org/doi/10.1103/PhysRevLett.14.973

[123] D. N. Nikogosyan, Nonlinear Optical Crystals: A Complete Survey: A Complete Survey, Springer Science \& Business Media, 2006.

1365

[124] V. Petrov, Parametric down-conversion devices: The coverage of the mid-infrared spectral range by solid-state laser sources, Optical Materials 34 (3) (2012) 536-554. doi:10.1016/j.optmat.2011.03.042.

1 URL http://linkinghub.elsevier.com/retrieve/pii/ S0925346711001674

1370 [125] C. McGowan, D. T. Reid, M. Ebrahimzadeh, W. Sibbett, Fem1. tosecond pulses tunable beyond $4 \mu \mathrm{m}$ from a KTA-based optical parametric oscillator, Optics Communications 134 (1997) 186 - 190. doi:http://dx.doi.org/10.1016/S0030-4018(96)00504-4.

[126] R. F. Wu, K. S. Lai, H. Wong, W.-J. Xie, Y. Lim, E. Lau, Multiwatt mid-IR output from a Nd:YALO laser pumped intracavity KTA OPO Opt. Express 8 (13) (2001) 694-698. doi:10.1364/0E.8.000694.

[127] K. Zhong, J. Yao, D. Xu, J. Wang, J. Li, P. Wang, High-pulse-energy a high-efficiency mid-infrared generation based on KTA optical paramet(1) ric oscillator, Applied Physics B 100 (4) (2010) 749-753. doi:10.1007/ s00340-010-3932-y.

$1385 \quad$ URL http://dx.doi.org/10.1007/s00340-010-3932-y 
[128] V. Petrov, F. Noack, Tunable femtosecond optical parametric amplifier in the mid-infrared with narrow-band seeding, J. Opt. Soc. Am. B 12 (11) (1995) 2214-2221. doi:10.1364/JOSAB.12.002214. URL http://josab.osa.org/abstract.cfm?URI=josab-12-11-2214

[129] G. R. Holtom, R. A. Crowell, X. S. Xie, High-repetition-rate femtosecond optical parametric oscillator-amplifier system near $3 \mu \mathrm{m}, \mathrm{J}$. Opt. Soc. Am. B 12 (9) (1995) 1723-1731. doi:10.1364/JOSAB.12.001723. URL http://josab.osa.org/abstract.cfm?URI=josab-12-9-1723

[130] B. Zysset, I. Biaggio, P. Günter, Refractive indices of orthorhombic KNbO3. I. Dispersion and temperature dependence, J. Opt. Soc. Am. B 9 (3) (1992) 380-386. doi:10.1364/JOSAB.9.000380. URL http://josab.osa.org/abstract.cfm?URI=josab-9-3-380

[131] I. Biaggio, B. Zysset, P. Kerkoc, L.-S. Wu, P. Günter, Refractive indices of orthorhombic KNbO3. II. Phase-matching configurations for nonlinearoptical interactions, J. Opt. Soc. Am. B 9 (4) (1992) 507-517. doi:10. 1364/JOSAB.9.000507.

URL http://josab. osa.org/abstract.cfm?URI=josab-9-4-507

[132] Y. Uematsu, Nonlinear Optical Properties of KNbO3 Single Crystal in the Orthorhombic Phase, Japanese Journal of Applied Physics 13 (9) (1974) 1362 URL http://stacks .iop. org/1347-4065/13/i=9/a=1362

[133] N. Umemura, K. Yoshida, K. Kato, Phase-matching properties of KNbO3 ․ in the mid-infrared, Appl. Opt. 38 (6) (1999) 991-994. doi:10.1364/AO. 38.000991 URL http://ao.osa.org/abstract.cfm?URI=ao-38-6-991

[134] J. A. Gruetzmacher, N. F. Scherer, Few-cycle mid-infrared pulse generation, characterization, and coherent propagation in optically dense media, Review of Scientific Instruments 73 (6) (2002) 2227-2236. doi:http://dx.doi.org/10.1063/1.1480464. URL http://scitation.aip.org/content/aip/journal/rsi/73/6/ $10.1063 / 1.1480464$

[135] C. J. Fecko, J. J. Loparo, A. Tokmakoff, Generation of 45 femtosecond pulses at $3 \mu \mathrm{m}$ with a $\{$ KNbO3\} optical parametric amplifier, Optics Communications 241 (2004) 521 - 528. doi:http://dx.doi.org/10.1016/j.optcom.2004.07.038.

II URL http://www.sciencedirect.com/science/article/pii/ S0030401804007576

[136] S. Cussat-Blanc, A. Ivanov, D. Lupinski, E. Freysz, KTiOPO4, KTiOAsO4, and KNbO3 crystals for mid-infrared femtosecond optical parametric amplifiers: analysis and comparison, Applied Physics B 70 (1) 
(2000) S247-S252. doi:10.1007/s003400000313.

URL http://dx.doi.org/10.1007/s003400000313

[137] S. Matsumoto, E. Lim, H. Hertz, M. Fejer, Quasiphase-matched second harmonic generation of blue light in electrically periodically-poled lithium tantalate waveguides, Electronics Letters 27 (22) (1991) 2040-2042. doi: 10.1049/el:19911263.

[138] K. Kato, High-power difference-frequency generation at 4.4-5.7 $\mu \mathrm{m}$ in LiIO3, Quantum Electronics, IEEE Journal of 21 (2) (1985) 119-120. doi:10.1109/JQE.1985.1072617.

1435 [139] U. Chatterjee, A. Rudra, G. Bhar, Generation of $6.8 \mu \mathrm{m}$ radiation in

1. lithium iodate, Applied Physics B 61 (5) (1995) 489-491. doi:10.1007/ BF01081278.

URL http://dx.doi.org/10.1007/BF01081278

[140] D. C. Hanna, V. V. Rampal, R. C. Smith, Tunable infrared downconversion in silver thiogallate, Optics Communications 8 (2) (1973) 151 - 153. doi:http://dx.doi.org/10.1016/0030-4018(73)90160-0. URL http://www.sciencedirect.com/science/article/pii/ 0030401873901600

[141] R. L. Byer, M. M. Choy, R. L. Herbst, D. S. Chemla, R. S. 1445 Feigelson, Second harmonic generation and infrared mixing in AgGaSe2, Applied Physics Letters 24 (2) (1974) 65-68. doi:http://dx.doi.org/10.1063/1.1655096.

11 URL http://scitation.aip.org/content/aip/journal/apl/24/2/ 10.1063/1.1655096

[142] H. Chou, R. Slater, Y. Wang, High-energy, fourth-harmonic generation using CO2 lasers, Applied Physics B 66 (5) (1998) 555-559. doi:10. $1007 / \mathrm{s} 003400050434$. URL http://dx.doi.org/10.1007/s003400050434

[143] G. D. Boyd, E. Buehler, F. G. Storz, Linear and nonlinear optical 1455 properties of ZnGeP2 and CdSe, Applied Physics Letters 18 (7) (1971) 301-304. doi:http://dx.doi.org/10.1063/1.1653673.

a URL http://scitation.aip.org/content/aip/journal/apl/18/7/ $10.1063 / 1.1653673$

[144] K. L. Vodopyanov, P. G. Schunemann, Broadly tunable noncritically 1460 phase-matched ZnGeP2 optical parametric oscillator with a $2-\mu \mathrm{J}$ pump threshold, Opt. Lett. 28 (6) (2003) 441-443. doi:10.1364/OL.28.000441. URL http://ol .osa.org/abstract .cfm?URI=ol-28-6-441

[145] A. Dergachev, D. Armstrong, A. Smith, T. Drake, M. Dubois, 3.4-um aGP RISTRA nanosecond optical parametric oscillator pumped by a 
[149] L. Isaenko, A. Yelisseyev, S. Lobanov, V. Petrov, F. Rotermund, G. Slekys, 【. J.-J. Zondy, LiInSe2: A biaxial ternary chalcogenide crystal for nonlinear 1485

[150] I. Pupeza, D. Sanchez, O. Pronin, J. Zhang, N. Lilienfein, M. Sei14413.

URL http://www.opticsinfobase.org/abstract.cfm?uri= oe-15-22-14404

[146] L. Kador, D. Haarer, K. Allakhverdiev, E. Salaev, Phase-matched secondharmonic generation at $789.5 \mathrm{~nm}$ in a GaSe crystal, Applied Physics Letters 69 (6) (1996) 731-733. doi:10.1063/1.117873.

[147] V. Petrov, A. Yelisseyev, L. Isaenko, S. Lobanov, A. Titov, J.-J. Zondy, Second harmonic generation and optical parametric amplification in the mid-IR with orthorhombic biaxial crystals LiGaS2 and LiGaSe2, Applied Physics B 78 (5) (2004) 543-546. doi:10.1007/s00340-004-1463-0. URL http://dx.doi .org/10.1007/s00340-004-1463-0

[148] L. Isaenko, A. Yelisseyev, S. Lobanov, V. Petrov, F. Rotermund, J.-J. Zondy, G. H. M. Knippels, LiInS2: A new nonlinear crystal for the mid-IR, Materials Science in Semiconductor Processing 4 (6) (2001) 665 -668. doi:http://dx.doi.org/10.1016/S1369-8001(02)00039-2. URL http://www.sciencedirect.com/science/article/pii/ S1369800102000392 (2002) 9475-9480. doi:http://dx.doi.org/10.1063/1.1478139. URL http://scitation.aip.org/content/aip/journal/jap/91/12/ $10.1063 / 1.1478139$

del, I. Znakovskaya, V. Pervak, Z. Wei, F. Krausz, A. Apolonski, J. Biegert, Compact 0.1-W Source of Octave-Spanning Mid-Infrared Femtosecond Pulses Centered at $10 \mu \mathrm{m}$, in: CLEO: 2014 Postdeadline Paper Digest, Optical Society of America, 2014, p. STh5C.7. doi:10.1364/CLEO_SI.2014.STh5C.7.

a URL http://www.opticsinfobase.org/abstract.cfm?URI=CLEO_ SI-2014-STh5C.7

[151] B. Levine, C. Bethea, H. Kasper, F. Thiel, Nonlinear optical susceptibility of HgGa2s4, Quantum Electronics, IEEE Journal of 12 (6) (1976) 367-368. doi:10.1109/JQE.1976.1069169

[152] V. V. Badikov, A. K. Don, K. V. Mitin, A. M. Seregin, V. V. Sinaiskii, N. I. Schebetova, A HgGa2s4 optical parametric oscillator, Quantum Electronics 33 (9) (2003) 831. URL http://stacks .iop.org/1063-7818/33/i=9/a=A12

[153] Cadmium silicon phosphide (CdSiP2) crystal structure, lattice parameters, thermal expansion, melting point, Ternary Compounds, Organic

2.05- $\mu \mathrm{m}$ Ho:YLF MOPA system, Optics express 15 (22) (2007) 14404- 
Semiconductors 41E (2000) 1-2.

URL http://dx.doi.org/10.1007/10717201_465

[154] V. Petrov, F. Noack, I. Tunchev, P. Schunemann, K. Zawilski, The nonlinear coefficient d36 of CdSiP2, Vol. 7197, 2009, pp. 71970M-71970M-8. doi : 10.1117/12.809586. URL http://dx.doi.org/10.1117/12.809586

[155] V. Petrov, P. G. Schunemann, K. T. Zawilski, T. M. Pollak, Noncritical singly resonant optical parametric oscillator operation near $6.2 \mu \mathrm{m}$ based on a CdSiP2 crystal pumped at $1064 \mathrm{~nm}$, Opt. Lett. 34 (16) (2009) 23992401. doi:10.1364/0L.34.002399.

URL http://ol .osa.org/abstract . cfm?URI=ol-34-16-2399

[156] D. Sánchez, M. Hemmer, M. Baudisch, K. Zawilski, P. Schunemann, H. Hoogland, R. Holzwarth, J. Biegert, Broadband mid-IR frequency comb with CdSiP2 and AgGaS2 from an Er,Tm:Ho fiber laser, Opt. Lett. 39 (24) (2014) 6883-6886. doi:10.1364/0L .39.006883. URL http://ol .osa.org/abstract . cfm?URI=ol-39-24-6883

[157] L. Z. Liu, K. O'Keeffe, S. M. Hooker, Quasi-phase-matching of high-orderharmonic generation using polarization beating in optical waveguides, Physical Review A 85 (5) (2012) 053823. doi:10.1103/PhysRevA.85. 053823.

URL http://link.aps .org/doi/10.1103/PhysRevA.85.053823

[158] H. Ishizuki, T. Taira, High-energy quasi-phase-matched optical parametric oscillation in a periodically poled $\mathrm{MgO}: \mathrm{LiNbO} 3$ device with a $5 \mathrm{~mm} \times 5$ mm aperture, Opt. Lett. 30 (21) (2005) 2918-2920. doi:10.1364/OL.30. 002918. URL http://ol.osa.org/abstract.cfm?URI=ol-30-21-2918

[159] H. Ishizuki, T. Taira, Half-joule output optical-parametric oscillation by using 10-mm-thick periodically poled Mg-doped congruent LiNbO3, Opt. Express 20 (18) (2012) 20002-20010. doi:10.1364/0E.20.020002. URL http://wWw.opticsexpress.org/abstract.cfm?URI= oe-20-18-20002

[160] K. L. Vodopyanov, E. Sorokin, I. T. Sorokina, P. G. Schunemann, Mid-IR

1. frequency comb source spanning 4.4-5.4 $\mu \mathrm{m}$ based on subharmonic GaAs optical parametric oscillator, Optics Letters 36 (12) (2011) 2275-2277. doi:10.1364/0L.36.002275. URL http://ol .osa.org/abstract .cfm?URI=ol-36-12-2275

[161] C. R. Phillips, J. Jiang, C. Mohr, A. C. Lin, C. Langrock, M. Snure, D. Bliss, M. Zhu, I. Hartl, J. S. Harris, M. E. Fermann, M. M.

a Fejer, Widely tunable midinfrared difference frequency generation in orientation-patterned GaAs pumped with a femtosecond Tm-fiber system, Optics Letters 37 (14) (2012) 2928. doi:10.1364/0L.37.002928 


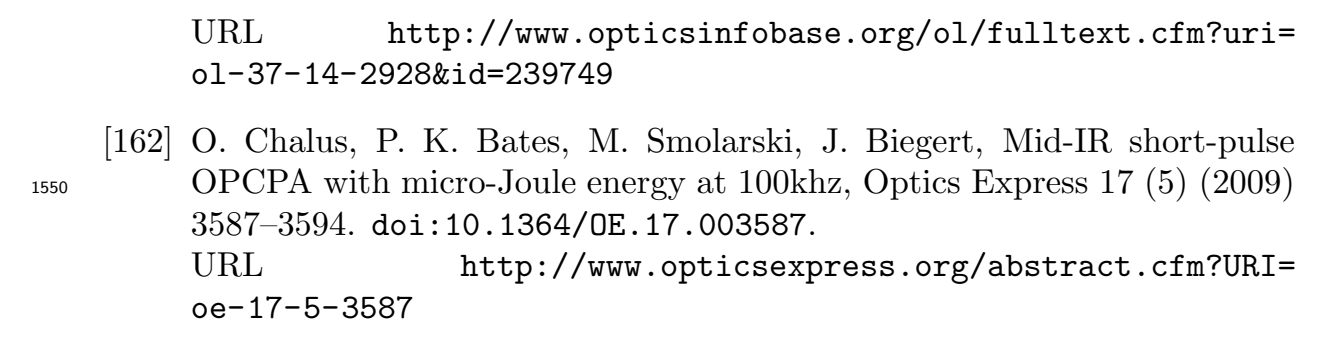

[163] D. Sanchez, M. Hemmer, M. Baudisch, H. Hoogland, R. Holzwarth, 1555 K. Zawilski, P. G. Schunemann, J. Biegert, Ultra-broadband DFG in a CdSiP2 at $6.5 \mu \mathrm{m}$ with 2.3 cycle transform limit from an Er:Tm:Ho fiber laser, in: CLEO: 2014, OSA Technical Digest (online), Optical Society of America, 2014, p. SM1I.1. doi:10.1364/CLEO_SI.2014.SM1I.1.

${ }_{1560}$ URL http://www.opticsinfobase.org/abstract.cfm?URI=CLEO_

[164] M. Beutler, I. Rimke, E. Büttner, V. Petrov, L. d. Isaenko, Difference-

a. frequency generation of fs and ps mid-IR pulses in LiInSe2 based on Yb-fiber laser pump sources, Optics Letters 39 (15) (2014) 4353-4355, cited By (since 1996)0.
1565 a URL http://www.opticsinfobase.org/ol/abstract.cfm?uri= ol-39-15-4353

[165] J. M. Fraser, D. Wang, A. Haché, G. R. Allan, H. M. v. Driel, Generation of high-repetition-rate femtosecond pulses from 8 to $18 \mu \mathrm{m}$, Appl. Opt. 36 (21) (1997) 5044-5047. doi:10.1364/A0.36.005044.

1570 URL http://ao.osa.org/abstract.cfm?URI=ao-36-21-5044

[166] M. Beutler, I. Rimke, E. Büttner, V. Badikov, D. Badikov, V. Petrov,

1. Efficient femtosecond $50 \mathrm{MHz}$ repetition rate mid-IR source up to $17 \mu \mathrm{m}$ by difference-frequency generation in AgGaSe2, in: Proceedings of SPIE - The International Society for Optical Engineering, Vol. 8964, 2014.
1575 URL http://www.scopus.com/inward/record.
url?eid=2-s2.0-84896761303\&partner ID=40\&md5= b9d51b5ce68c5c5603581e67a023aaa8

[167] S. Ehret, H. Schneider, Generation of subpicosecond infrared pulses tunable between $5.2 \mu \mathrm{m}$ and $18 \mu \mathrm{m}$ at at repetition rate of $76 \mathrm{MHz}$, Applied

${ }_{1580} \quad$ Physics B 66 (1) (1998) 27-30. doi:10.1007/s003400050352. URL http://dx .doi .org/10.1007/s003400050352

[168] F. Zernike, P. R. Berman, Generation of Far Infrared as a Difference घ. Frequency, Phys. Rev. Lett. 15 (26) (1965) 999-1001. doi:10.1103/ PhysRevLett.15.999.

1585 URL http://link.aps.org/doi/10.1103/PhysRevLett.15.999

[169] M. R. X. d. Barros, P. C. Becker, Two-color synchronously mode-locked 1. femtosecond Ti:sapphire laser, Opt. Lett. 18 (8) (1993) 631-633. doi: 
10.1364/OL.18.000631.

URL http://ol.osa.org/abstract . cfm?URI=ol-18-8-631

1590 [170] T. M. Jedju, L. Rothberg, Tunable femtosecond radiation in the midinfrared for time-resolved absorption in semiconductors, Appl. Opt. 27 (3) (1988) 615-618. doi:10.1364/A0.27.000615. URL http: //ao.osa.org/abstract .cfm?URI=ao-27-3-615

[171] U. Keller, T. Südmeyer, Ultrafast Laser Oscillators in the Thin Disk

1595 Geometry, Optik \& Photonik 3 (4) (2008) 39-44. doi:10.1002/opph. 201190221. URL http://dx.doi.org/10.1002/opph.201190221

[172] C. J. Saraceno, C. Schriber, F. Emaury, O. H. Heckl, C. R. E. Baer, M. Hoffmann, K. Beil, C. Kränkel, M. Golling, T. Südmeyer, U. Keller, Cutting-Edge High-Power Ultrafast Thin Disk Oscillators, Applied Sciences 3 (2) (2013) 355-395. doi:10.3390/app3020355.

URL http://www .mdpi .com/2076-3417/3/2/355

[173] F. P. Druon, S. Ricaud, A. Jaffre, K. Wentsch, A. Suganuma, B. Viana, P. Loiseau, B. Weichelt, M. A. Ahmed, A. Voss, T. Graf, D. Rytz, C. Hönninger, E. Mottay, M. Hanna, P. Georges, High power Yb:CALGO thin-disk lasers in cw and fs regime, in: CLEO: 2013, Optical Society of America, 2013, p. CTu1O.2. doi:10.1364/CLEO_SI.2013.CTu10.2.

a URL http://www.opticsinfobase.org/abstract.cfm?URI=CLEO_ SI-2013-CTu10.2

[174] B. Dannecker, X. Délen, K. S. Wentsch, B. Weichelt, C. Hönninger, A. Voss, M. A. Ahmed, T. Graf, Passively mode-locked Yb:CaF2 thin-disk laser, Opt. Express 22 (19) (2014) 22278-22284. doi:10.1364/OE.22.022278.

1 URL http://www.opticsexpress.org/abstract.cfm?URI= $1615 \quad$ oe-22-19-22278

[175] C. J. Saraceno, F. Emaury, C. Schriber, M. Hoffmann, M. Golling, T. Südmeyer, U. Keller, Ultrafast thin-disk laser with $80 \mu \mathrm{J}$ pulse en(1) ergy and $242 \mu \mathrm{W}$ of average power, Opt. Lett. 39 (1) (2014) 9-12. doi: 10.1364/0L.39.000009.

1620 URL http://ol.osa.org/abstract.cfm?URI=ol-39-1-9

[176] F. M. Mitschke, L. F. Mollenauer, Discovery of the soliton self-frequency shift, Opt. Lett. 11 (10) (1986) 659-661. doi:10.1364/0L.11.000659. URL http://ol .osa.org/abstract . cfm?URI=ol-11-10-659

[177] A. Gouveia-Neto, A. Gomes, J. Taylor, Femto soliton Raman generation, 1625 Quantum Electronics, IEEE Journal of 24 (2) (1988) 332-340. doi:10. $1109 / 3.130$ 
[178] D. G. Winters, P. Schlup, R. A. Bartels, Subpicosecond fiber-based solitontuned mid-infrared source in the 9.7-14.9 $\mu \mathrm{m}$ wavelength region, Opt. Lett. 35 (13) (2010) 2179-2181. doi:10.1364/OL .35.002179. URL http://ol .osa.org/abstract .cfm?URI=ol-35-13-2179

[179] T. W. Neely, T. A. Johnson, S. A. Diddams, High-power broadband laser source tunable from $3.0 \mu \mathrm{m}$ to $4.4 \mu \mathrm{m}$ based on a femtosecond Yb:fiber oscillator, Opt. Lett. 36 (20) (2011) 4020-4022. doi:10.1364/OL.36. 004020 .

URL http: //ol .osa.org/abstract . cfm?URI=ol-36-20-4020

[180] A. A. Lanin, A. B. Fedotov, A. M. Zheltikov, Ultrabroadband XFROG of few-cycle mid-infrared pulses by four-wave mixing in a gas, J. Opt. Soc. Am. B 31 (8) (2014) 1901-1905. doi:10.1364/JOSAB.31.001901. URL http://josab.osa.org/abstract.cfm?URI=josab-31-8-1901

1640 [181] H. Suchowski, D. Oron, A. Arie, Y. Silberberg, Geometrical representation of sum frequency generation and adiabatic frequency conversion, Phys. Rev. A 78 (6) (2008) 063821. doi:10.1103/PhysRevA.78.063821. URL http://link .aps .org/doi/10.1103/PhysRevA.78.063821

[182] J. Moses, F. X. Kaertner, H. Suchowski, Fully Efficient Adiabatic Down-

1645 conversion of Broadband Ti:sapphire Oscillator Pulses, in: Conference on Lasers and Electro-Optics 2012, Optical Society of America, 2012, p. CTh3B.3. doi:10.1364/CLEO_SI.2012.CTh3B.3.

11 URL http://www.opticsinfobase.org/abstract.cfm?URI=CLEO_ SI-2012-CTh3B.3

[183] J. Moses, P. R. Krogen, S.-W. Huang, F. X. Kaertner, H. Suchowski, a Octave-spanning Coherent Mid-IR Pulses via Adiabatic Difference Frequency Generation, in: Nonlinear Optics, Optical Society of America, 2013, p. NF1A.6. doi:10.1364/NLO.2013.NF1A.6.

1 URL http://www.opticsinfobase.org/abstract.cfm?URI= NLO-2013-NF1A.6

[184] L. Myers, W. Bosenberg, Periodically poled lithium niobate and quasiphase-matched optical parametric oscillators, Quantum Electronics, IEEE Journal of 33 (10) (1997) 1663-1672. doi:10.1109/3.631262.

185] J. Raffy, T. Debuisschert, J.-P. Pocholle, M. Papuchon, Tunable IR laser source with optical parametric oscillators in series, Appl. Opt. 33 (6) (1994) 985-987. doi:10.1364/A0.33.000985. URL http://ao.osa.org/abstract.cfm?URI=ao-33-6-985

[186] P. B. Phua, K. S. Lai, R. F. Wu, T. C. Chong, High-efficiency mida. infrared ZnGeP2 optical parametric oscillator in a multimode-pumped 1665 tandem optical parametric oscillator, Appl. Opt. 38 (3) (1999) 563-565. doi:10.1364/A0.38.000563. URL http://ao.osa.org/abstract.cfm?URI=ao-38-3-563 
[187] S. Chaitanya Kumar, A. Esteban-Martin, T. Ideguchi, M. Yan, S. Holzner, T. W. Hänsch, N. Picqué, M. Ebrahim-Zadeh, Few-cycle, broadband, mid$1670 \quad$ infrared optical parametric oscillator pumped by a 20-fs Ti:sapphire laser

1. Laser \& Photonics Reviews 8 (5) (2014) L86-L91. doi:10.1002/lpor. 201400091.

URL http://dx.doi .org/10.1002/lpor.201400091

[188] M. W. Haakestad, H. Fonnum, G. Arisholm, E. Lippert, K. Stenersen, 1675 a Mid-infrared optical parametric oscillator synchronously pumped by an erbium-doped fiber laser, Opt. Express 18 (24) (2010) 25379-25388. doi:10.1364/OE.18.025379.

a URL http://www.opticsexpress.org/abstract.cfm?URI= oe-18-24-25379

[189] F. Adler, K. C. Cossel, M. J. Thorpe, I. Hartl, M. E. Fermann, J. Ye, Phase-stabilized, $1.5 \mathrm{~W}$ frequency comb at 2.8-4.8 $\mu \mathrm{m}$, Opt. Lett. 34 (9) (2009) 1330-1332. doi:10.1364/0L.34.001330.

URL http://ol .osa.org/abstract.cfm?URI=ol-34-9-1330

[190] N. Leindecker, A. Marandi, R. L. Byer, K. L. Vodopyanov, J. Jiang, 1685 I. Hartl, M. Fermann, P. G. Schunemann, Octave-spanning ultra-

fast OPO with 2.6-6.1 $\mu \mathrm{m}$ instantaneous bandwidth pumped by femtosecond Tm-fiber laser, Opt. Express 20 (7) (2012) 7046-7053. doi:10.1364/OE.20.007046. URL http://www.opticsexpress.org/abstract.cfm?URI= oe-20-7-7046

[191] Z. Zhang, D. T. Reid, S. C. Kumar, M. Ebrahim-Zadeh, P. G. Schunemann, K. T. Zawilski, C. R. Howle, Femtosecond-laser pumped CdSiP2 optical parametric oscillator producing $100 \mathrm{MHz}$ pulses centered at 6.2 $\mu \mathrm{m}$, Opt. Lett. 38 (23) (2013) 5110-5113. doi:10.1364/0L.38.005110. URL http://ol .osa.org/abstract.cfm?URI=ol-38-23-5110

[192] N. Hendaoui, A. Peremans, P. G. Schunemann, K. T. Zawilski, V. Petrov, 1. Synchronously pumped OPO for picosecond pulse generation in the midinfrared near $6.45 \mu \mathrm{m}$ using AgGaS2 and CdSiP2: a comparative study,

1. Laser Physics 23 (8) (2013) 085401. doi:10.1088/1054-660X/23/8/ 085401 .

URL http://iopscience.iop.org/1555-6611/23/8/085401

[193] P. A. Budni, L. A. Pomeranz, M. L. Lemons, P. G. Schunemann, T. M. Pollak, E. P. Chicklis, 10w Mid-IR Holmium Pumped ZnGeP2 OPO, in: Advanced Solid State Lasers, Optical Society of America, 1998, p. FC1. doi:10.1364/ASSL.1998.FC1.

1. URL http://www.opticsinfobase.org/abstract.cfm?URI= ASSL-1998-FC1

[194] K. L. Vodopyanov, F. Ganikhanov, J. P. Maffetone, I. Zwieback, W. Ruderman, ZnGeP2 optical parametric oscillator with 3.8-12.4 $\mu \mathrm{m}$ tunability, 
Opt. Lett. 25 (11) (2000) 841-843. doi:10.1364/0L. 25.000841.

URL http: //ol .osa.org/abstract . cfm?URI=ol-25-11-841

[195] D. Creeden, M. Jiang, P. A. Budni, P. A. Ketteridge, S. D. Setzler, Y. E. Young, J. C. McCarthy, P. G. Schunemann, T. M. Pollak, P. Tayebati, E. P. Chicklis, Thulium fiber laser-pumped mid-IR OPO, Vol. 6952, 2008, pp. 69520S-69520S-7. doi:10.1117/12.775196. URL http://dx.doi.org/10.1117/12.775196

[196] E. Lippert, H. Fonnum, G. Arisholm, K. Stenersen, A 22-

w watt mid-infrared optical parametric oscillator with V-shaped 3mirror ring resonator, Opt. Express 18 (25) (2010) 26475-26483. doi:10.1364/OE.18.026475. URL oe-18-25-26475

[197] G. Stoeppler, N. Thilmann, V. Pasiskevicius, A. Zukauskas, C. Canalias, M. Eichhorn, Tunable Mid-infrared ZnGeP2 RISTRA OPO 1725 pumped by periodically-poled Rb:KTP optical parametric masteroscillator power amplifier, Opt. Express 20 (4) (2012) 4509-4517. doi:10.1364/OE.20.004509.

a URL http://www.opticsexpress.org/abstract.cfm?URI= oe-20-4-4509

1730 [198] M. Haakestad, H. Fonnum, E. Lippert, Mid-infrared source with 0.2 1. J pulse energy based on nonlinear conversion of Q-switched pulses in ZnGeP2, Optics Express 22 (7) (2014) 8556-8564.

I. URL http://www.scopus.com/inward/record.

11 url?eid=2-s2.0-84898645110\&partnerID=40\&md5=

$1735 \quad$ aca566573863137a5938d05376d2b92d

[199] M. Schellhorn, G. Stoeppler, S. Bigotta, A. Hildenbrand, C. Kieleck, M. Eichhorn, High-pulse energy mid-IR ZGP OPO, in: Advanced SolidState Lasers Congress, Optical Society of America, 2013, p. MW3B.1. doi:10.1364/MICS.2013.MW3B.1.

1740 प URL http://www.opticsinfobase.org/abstract.cfm?URI= MICS-2013-MW3B.1

[200] F. Silva, P. K. Bates, A. Esteban-Martin, M. Ebrahim-Zadeh, J. Biegert, High-average-power, carrier-envelope phase-stable, few-cycle pulses at 2.1 $\mu \mathrm{m}$ from a collinear BiB3o6 optical parametric amplifier, Opt. Lett. 37 (5) (2012) 933-935. doi:10.1364/0L.37.000933 URL http: //ol .osa .org/abstract . cfm?URI=ol-37-5-933

[201] F. Seifert, V. Petrov, M. Woerner, Solid-state laser system for the generation of midinfrared femtosecond pulses tunable from 3.3 to $10 \mu \mathrm{m}$, Opt. Lett. 19 (23) (1994) 2009-2011. doi:10.1364/0L.19.002009. URL http://ol .osa.org/abstract.cfm?URI=ol-19-23-2009 
[202] A. Sugiharto, C. Johnson, H. de Aguiar, L. Alloatti, S. Roke, Generation and application of high power femtosecond pulses in the vibrational fingerprint region, Applied Physics B 91 (2) (2008) 315-318. doi:10.1007/s00340-008-2993-7 URL http://dx.doi .org/10.1007/s00340-008-2993-7

[203] H.-T. Fan, C.-H. Xu, Z.-H. Wang, G. Wang, C.-J. Liu, J.-K. Liang, X.-L. Chen, Z.-Y. Wei, Generation of broadband $17 \mu \mathrm{J}$ mid-infrared femtosecond pulses at $3.75 \mu \mathrm{m}$ by silicon carbide crystal, Opt. Lett. 39 (21) (2014) 6249-6252. doi:10.1364/0L.39.006249. URL http://ol .osa.org/abstract . cfm?URI=ol-39-21-6249

[204] G. Andriukaitis, T. Balčiūnas, S. Ališauskas, A. Pugžlys, A. Baltuška, T. Popmintchev, M.-C. Chen, M. M. Murnane, H. C. Kapteyn, 90 GW peak power few-cycle mid-infrared pulses from an optical parametric amplifier, Opt. Lett. 36 (15) (2011) 2755-2757. doi:10.1364/0L .36.002755. URL http://ol.osa.org/abstract.cfm?URI=ol-36-15-2755

[205] M. Hemmer, A. Thai, M. Baudisch, H. Ishizuki, T. Taira, J. Biegert, 18uJ energy, $160-\mathrm{kHz}$ repetition rate, 250-MW peak power mid-IR OPCPA, Chinese Optics Letters 11 (1) (2013) 013202. URL http://col.osa.org/abstract.cfm?URI=col-11-1-013202

1770 [206] K. Zhao, H. Zhong, P. Yuan, G. Xie, J. Wang, J. Ma, L. Qian, Generation a. of $120 \mathrm{GW}$ mid-infrared pulses from a widely tunable noncollinear optical 1 parametric amplifier, Opt. Lett. 38 (13) (2013) 2159-2161. doi:10.1364/ OL.38.002159. URL http://ol.osa.org/abstract .cfm?URI=ol-38-13-2159

1775 [207] B. W. Mayer, C. R. Phillips, L. Gallmann, U. Keller, Mid-infrared pulse generation via achromatic quasi-phase-matched OPCPA, Opt. Express 22 (17) (2014) 20798-20808. doi:10.1364/OE.22.020798

1 URL http://www.opticsexpress.org/abstract.cfm?URI= oe-22-17-20798

${ }_{1780}$ [208] M. Gerrity, S. Brown, T. Popmintchev, M. Murnane, H. Kapteyn, a S. Backus, High Repetition Rate, mJ-Level, mid-IR OPCPA System, in: CLEO: 2014, Optical Society of America, 2014, p. STh4E.8. doi:10.1364/CLEO_SI.2014.STh4E.8.

1 URL http://www.opticsinfobase.org/abstract.cfm?URI=CLEO_ $1785 \quad$ SI-2014-STh4E.8

[209] S. L. Cousin, F. Silva, S. Teichmann, M. Hemmer, B. Buades, J. Biegert, High-flux table-top soft x-ray source driven by sub-2-cycle, CEP stable, $1.85-\mu \mathrm{m} \mathrm{1-kHz}$ pulses for carbon K-edge spectroscopy, Opt. Lett. 39 (18) (2014) 5383-5386. doi:10.1364/OL . 39.005383. URL http://ol.osa.org/abstract.cfm?URI=ol-39-18-5383 
[210] A. Baltuška, T. Fuji, T. Kobayashi, Controlling the Carrier-Envelope Phase of Ultrashort Light Pulses with Optical Parametric Amplifiers,

n Phys. Rev. Lett. 88 (13) (2002) 133901. doi:10.1103/PhysRevLett.88. 133901 . URL http://link.aps .org/doi/10.1103/PhysRevLett.88.133901

[211] M. Hemmer, D. Sanchez, M. Jelínek, V. Smirnov, H. Jelinkova, V. Kubeček, J. Biegert, $2 \mu \mathrm{m}$ wavelength, high energy Ho:YLF chirped pulse amplifier for mid-infrared OPCPA,, To be published.

[212] B. J. Eggleton, B. Luther-Davies, K. Richardson, Chalcogenide photonics Nat Photon 5 (3) (2011) 141-148. doi:10.1038/nphoton.2011.309. URL http://dx.doi.org/10.1038/nphoton.2011.309

[213] D. D. Hudson, M. Baudisch, D. Werdehausen, B. J. Eggleton, J. Biegert, 1.9 octave supercontinuum generation in a As2s3 step-index fiber driven by mid-IR OPCPA, Opt. Lett. 39 (19) (2014) 5752-5755. doi:10.1364/ OL. 39.005752, URL http://ol.osa.org/abstract.cfm?URI=ol-39-19-5752

[214] Y. Yu, X. Gai, T. Wang, P. Ma, R. Wang, Z. Yang, D.-Y. Choi, S. Madden, B. Luther-Davies, Mid-infrared supercontinuum generation in chalcogenides, Opt. Mater. Express 3 (8) (2013) 1075-1086. doi:10.1364/OME.3.001075.

1 URL http://www.opticsinfobase.org/ome/abstract.cfm?URI= ome-3-8-1075

[215] I. Kubat, C. S. Agger, U. Møller, A. B. Seddon, Z. Tang, S. Sujecki, T. M. Benson, D. Furniss, S. Lamrini, K. Scholle, P. Fuhrberg, B. Napier, 1815 M. Farries, J. Ward, P. M. Moselund, O. Bang, Mid-infrared super1. continuum generation to $12.5 \mu \mathrm{m}$ in large NA chalcogenide step-index fibres pumped at $4.5 \mu \mathrm{m}$, Opt. Express 22 (16) (2014) 19169-19182. doi:10.1364/OE.22.019169. URL http://www.opticsexpress.org/abstract.cfm?URI= oe-22-16-19169

[216] M. Poulain, M. Poulain, J. Lucas, Verres fluores au tetraflua orure de zirconium proprietes optiques d'un verre dope au Nd3+, Materials Research Bulletin 10 (4) (1975) 243 - 246. doi:http://dx.doi.org/10.1016/0025-5408(75)90106-3.

1825 URL http://wWW.sciencedirect.com/science/article/pii/ 0025540875901063

[217] K. Ohsawa, T. Shibata, Preparation and characterization of ZrF4-BaF2LaF3-NaF-AIF3 glass optical fibers, Lightwave Technology, Journal of 2 (5) (1984) 602-606. doi:10.1109/JLT.1984.1073686.

${ }_{1830}$ [218] O. P. Kulkarni, V. V. Alexander, M. Kumar, M. J. Freeman, M. N. Islam, घ. J. Fred L. Terry, M. Neelakandan, A. Chan, Supercontinuum generation 
[221] A. M. Heidt, J. H. V. Price, C. Baskiotis, J. S. Feehan, Z. Li, S. U. Alam,

\section{[222]}

[225] M. Nisoli, S. De Silvestri, O. Svelto, Generation of high energy 10 fs pulses by a new pulse compression technique, Applied Physics Letters 68 (20) (1996) 2793-2795. doi:http://dx.doi.org/10.1063/1.116609.

n URL http://scitation.aip.org/content/aip/journal/apl/68/20/

$10.1063 / 1.116609$

URL http://josab.osa.org/abstract.cfm?URI=josab-28-10-2486

219] J. Swiderski, M. Michalska, High-power supercontinuum generation in a ZBLAN fiber with very efficient power distribution toward the midinfrared, Optics Letters 39 (4) (2014) 910-913. doi:10.1364/OL.39. 000910 .

URL http://ol.osa.org/abstract.cfm?URI=ol-39-4-910

220] C. Xia, M. Kumar, O. P. Kulkarni, M. N. Islam, J. Fred L. Terry, M. J. Freeman, M. Poulain, G. Mazé, Mid-infrared supercontinuum generation to $4.5 \mu \mathrm{m}$ in ZBLAN fluoride fibers by nanosecond diode pumping, Opt. Lett. 31 (17) (2006) 2553-2555. doi:10.1364/0L.31.002553. URL http://ol .osa.org/abstract .cfm?URI=ol-31-17-2553

D. J. Richardson, Mid-infrared ZBLAN fiber supercontinuum source using picosecond diode-pumping at $2 \mu \mathrm{m}$, Opt. Express 21 (20) (2013) 24281-24287. doi:10.1364/OE.21.024281.

oe-21-20-24281 http://www.opticsexpress.org/abstract.cfm?URI= generated in a fluoride fiber pumped by $\mathrm{Er} \& \mathrm{Er}$ :Yb-doped and Tmdoped fiber amplifiers, Optics \& Laser Technology 52 (0) (2013) $75-80$. doi:http://dx.doi.org/10.1016/j.optlastec.2013.04.013 URL http://www.sciencedirect.com/science/article/pii/ S0030399213001394

mid-infrared supercontinuum generation in a single mode ZBLAN fiber pumped by a $2 \mu \mathrm{m}$ MOPA system, Opt. Lett. 39 (7) (2014) 1849-1852. doi:10.1364/OL.39.001849.

URL http://ol.osa.org/abstract.cfm?URI=ol-39-7-1849

[224] A. Couairon, A. Mysyrowicz, Femtosecond filamentation in transparent media, Physics Reports 441 (2-4) $(2007) \quad 47$ - 189. doi:http://dx.doi.org/10.1016/j.physrep.2006.12.005. URL http://www.sciencedirect.com/science/article/pii/ S037015730700021X

from 1.9 to $4.5 \mu \mathrm{m}$ in ZBLAN fiber with high average power generation beyond $3.8 \mu \mathrm{m}$ using a thulium-doped fiber amplifier, J. Opt. Soc. Am. B 28 (10) (2011) 2486-2498. doi:10.1364/JOSAB . 28.002486. 
[226] C. Hauri, W. Kornelis, F. Helbing, A. Heinrich, A. Couairon, A. Mysyrowicz, J. Biegert, U. Keller, Generation of intense, carrier-envelope phaselocked few-cycle laser pulses through filamentation, Applied Physics B 79 (6) (2004) 673-677. doi:10.1007/s00340-004-1650-z. URL http://dx.doi.org/10.1007/s00340-004-1650-z

[227] A. Verhoef, J. Seres, K. Schmid, Y. Nomura, G. Tempea, L. Veisz, F. Krausz, Compression of the pulses of a Ti:sapphire laser system to 5 femtoseconds at 0.2 terawatt level, Applied Physics B 82 (4) (2006) 513-517. doi:10.1007/s00340-005-2104-y. URL http://dx.doi .org/10.1007/s00340-005-2104-y

[228] B. Schenkel, J. Biegert, U. Keller, C. Vozzi, M. Nisoli, G. Sansone, S. Stagira, S. D. Silvestri, O. Svelto, Generation of 3.8-fs pulses from adaptive compression of a cascaded hollow fiber supercontinuum Opt. Lett. 28 (20) (2003) 1987-1989. doi:10.1364/0L.28.001987. URL http://ol.osa.org/abstract .cfm?URI=ol-28-20-1987

[229] A. A. Lanin, A. A. Voronin, E. A. Stepanov, A. B. Fedotov, A. M. Zheltikov, Frequency-tunable sub-two-cycle 60-MW-peak-power freespace waveforms in the mid-infrared, Opt. Lett. 39 (22) (2014) 6430-6433. doi:10.1364/OL.39.006430. URL http://ol .osa.org/abstract.cfm?URI=ol-39-22-6430

[230] C. P. Hauri, R. B. Lopez-Martens, C. I. Blaga, K. D. Schultz, J. Cryan, R. Chirla, P. Colosimo, G. Doumy, A. M. March, C. Roedig, E. Sistrunk, 1895 J. Tate, J. Wheeler, L. F. DiMauro, E. P. Power, Intense self-compressed, self-phase-stabilized few-cycle pulses at $2 \mu \mathrm{m}$ from an optical filament, Opt. Lett. 32 (7) (2007) 868-870. doi:10.1364/0L.32.000868. URL http://ol .osa.org/abstract.cfm?URI=ol-32-7-868

[231] L. Bergé, J. Rolle, C. Köhler, Enhanced self-compression of mid-infrared $1900 \quad$ laser filaments in argon, Phys. Rev. A 88 (2) (2013) 023816. doi:10. 1103/PhysRevA.88.023816. URL http://link.aps.org/doi/10.1103/PhysRevA.88.023816

[232] F. Silva, D. Austin, A. Thai, M. Baudisch, M. Hemmer, D. Faccio, A. Couairon, J. Biegert, Multi-octave supercontinuum generation from mid-infrared filamentation in a bulk crystal, Nature Communications 3 (2012) 807. doi:10.1038/ncomms1816. URL http://www . nature.com/doifinder/10.1038/ncomms1816

[233] M. Hemmer, M. Baudisch, A. Thai, A. Couairon, J. Biegert, Self-compression to sub-3-cycle duration of mid-infrared optical ${ }_{1910} \quad$ pulses in dielectrics, Opt. Express 21 (23) (2013) 28095-28102. doi:10.1364/0E.21.028095.

URL http://www.opticsexpress.org/abstract.cfm?URI= oe-21-23-28095 
[234] A. Pugzlys, P. Malevich, S. Alisauskas, A. A. Voronin, D. Kar1915 tashov, A. Baltuska, A. Zheltikov, D. Faccio, Self-compression of Millijoule mid-IR Femtosecond Pulses in Transparent Dielectrics, in: CLEO: 2014, Optical Society of America, 2014, p. FTh1D.3. doi:10.1364/CLEO_QELS.2014.FTh1D.3

URL http://www.opticsinfobase.org/abstract.cfm?URI=CLEO_ 1920 QELS-2014-FTh1D.3 